CERN-TH/97-360

US-FT-34/97

hep-th/9712139

\title{
Gauge-Invariant Operators for Singular Knots in Chern-Simons Gauge Theory
}

\author{
J. M. F. Labastida ${ }^{a, b}$ and Esther Pérez ${ }^{b}$ \\ a Theory Division, CERN, \\ CH-1211 Geneva 23, Switzerland. \\ ${ }^{b}$ Departamento de Física de Partículas, \\ Universidade de Santiago de Compostela, \\ E-15706 Santiago de Compostela, Spain.
}

\begin{abstract}
We construct gauge invariant operators for singular knots in the context of Chern-Simons gauge theory. These new operators provide polynomial invariants and Vassiliev invariants for singular knots. As an application we present the form of the Kontsevich integral for the case of singular knots.
\end{abstract}

CERN-TH/97-360

December 1997 


\section{Introduction}

Chern-Simons gauge theory [1] has shown to be a very powerful tool to study knot and links invariants. Its analysis from both, the perturbative and the non-perturbative points of view has provided numerous important insights in the theory of these invariants. Non-perturbative methods [1, 2, 3, 4, 5, 6] have established the connection of Chern-Simons gauge theory with polynomial invariants as the Jones polynomial [7] and its generalizations [8, 9, 10]. Perturbative methods [11, 12, 13, 14, 15, 16, 17] have provided representations of Vassiliev invariants.

In the context of Chern-Simons gauge theory, knot and link invariants have been obtained studying the vacuum expectation values of the associated Wilson loops, or product of Wilson loops. Since these loops correspond to knots or components of links, there are no intersections and the vacuum expectation values are well defined. Indeed, these vacuum expectation values turn out to be knot and link invariants. The story is different if one considers vacuum expectation values of Wilson loops or products of Wilson loops with intersections. The presence of singularities complicates the analysis and it is not known if they can be defined consistently. Fortunately, these objects are not the ones in which one is interested in the study of singular knots and links from the point of view of the theory of Vassiliev invariants. In this theory one must regard the invariant associated to a knot or link with an intersection as a certain difference of the invariants which correspond to different ways of solving the intersection. For example, if one considers the case of knots with a double point, invariants associated to the singular knot are defined as a difference between the invariant associated to the two resolutions of the double point (overcrossing and undercrossing). Invariants for singular knots with more than one double point are defined following an iterative procedure. The problem which we address in this paper is the construction of the gauge invariant operators associated to singular knots when their invariants are defined as differences of invariants involving singular knots with one less double point.

Singular knots have been studied in the context of Chern-Simons gauge theory to build physical states of quantum gravity in the Ashtekar formalism [18]. Our work can be regarded in part as a development of the approach to invariants for singular knots initiated in [18]. Singular knots have been also considered in other works [19, 20], as they appear as intermediate knot con- 
figurations in the study of non-singular knot invariants from a perturbative point of view. In the present work the emphasis is focused on the study of the singular knots themselves and on the construction of a generalization of the Wilson loop which leads to singular knots invariants satisfying the features imposed by the theory of Vassiliev invariants.

Our construction is based on functional integral technics. It involves the use of standard formulae for the variation a Wilson line under a deformation of its path. We will analyze families of singular knots parametrized by a parameter $u$ such that for $u=0$ there occurs a self-intersection, a double point, at some point $P \in M$, where $M$ is some boundaryless smooth threemanifold. For $u \neq 0$ there are not self-intersections in a neighborhood of $P$. If the singular knots of a given family are parametrized by a parameter $v$, two values of this parameter, $v=s$ and $v=t$, with $s \neq t$, correspond to the point $P$ for $u=0$. All singular knots with $u>0$ are equivalent under rigid vertex isotopy. The same property holds for all the ones with $u<0$. The difference between the two sets is that an undercrossing near $P$ is replaced by an overcrossing. A review on the basic concepts involved in the theory of singular knots can be found in [21]. If we denote by $\psi_{+}$the invariant associated to the singular knot for $u>0$, and by $\psi_{-}$the one for $u<0$, the invariant associated to the singular knot with one more double point is defined as $\psi_{+}-\psi_{-}$. In our construction we will analyze this difference computing the first derivative respect to $u$ of the vacuum expectation value of the operator associated to the family of singular knots. As a function of $u$ such a vacuum expectation value behaves as a step function around $u=0$. We will indeed find that its first derivative respect to $u$ has a delta-function behavior. Its integration along a finite interval enclosing $u=0$ is then carried out very simply, providing the searched operators whose vacuum expectation values lead to $\psi_{+}-\psi_{-}$.

The form of the new operators turn out to be very simple. Let us consider a singular oriented knot $K^{n}$ (parametrized by a parameter $v$ ) with $n$ singularities, all of them consisting of double points. These occur at points $P_{i}$, $i=1, \ldots, n$, in $M$. Let us choose as base point one of them and let us denote by $s_{i}, t_{i}$, with $s_{i}<t_{i}$, for $i=1, \ldots, n$, the values of the parameter $v$ at the double points. In addition, to each double point we also associate a group generator whose group index is labeled as $T^{a_{i}}, i=1, \ldots, n$. Thus, at each double point $P_{i}$ we have a triple $\tau_{i}=\left\{s_{i}, t_{i}, T^{a_{i}}\right\}$. Traveling along the knot starting from the base point the parameter $v$ takes the value $s_{i}$ when one 


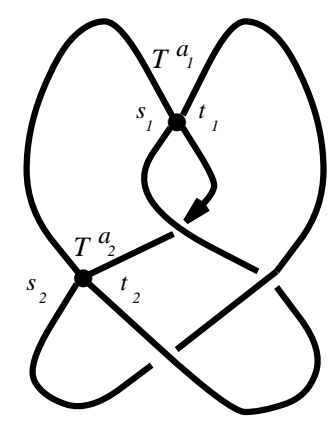

Figure 1: Example of a singular knot with two double points.

encounters the singular point $P_{i}$ for first time, and $t_{i}$ when one encounters it a second time. All the elements of the set $\left\{s_{i}, t_{i} ; i=1, \ldots, n\right\}$ are different. We can therefore order them into $\left\{w_{1}, w_{2}, \ldots, w_{n}\right\}$ with $w_{j}<w_{j+1}$. Each $w_{j}$ belongs to one and only one of the triples $\tau_{i}$. This order induces a mapping $\phi\left(w_{j}\right)$ which assigns to $w_{j}$ the index of the group generator in the triple to which $w_{j}$ belongs. The gauge invariant operator associated to the singular knot $K^{n}$ has the form:

$$
\begin{gathered}
(2 x)^{n} \operatorname{Tr}\left[T^{\phi\left(w_{1}\right)} U\left(w_{1}, w_{2}\right) T^{\phi\left(w_{2}\right)} U\left(w_{2}, w_{3}\right) T^{\phi\left(w_{3}\right)} \ldots\right. \\
\left.\cdots U\left(w_{2 n-1}, w_{2 n}\right) T^{\phi\left(w_{2 n}\right)} U\left(w_{2 n}, w_{1}\right)\right],
\end{gathered}
$$

where,

$$
x=\frac{2 \pi i}{k}
$$

and $U\left(w_{j}, w_{j+1}\right)$ denotes the Wilson line along the path joining the points corresponding to $v=w_{j}$ and $v=w_{j+1}$. Notice that the mapping $\phi$ is two to one and therefore there are not free group indices. Notice also that (1.1) does not depend on the choice of base point since it is invariant under cyclic permutations of the singular points.

The structure of the operators (1.1) allows to classify the singular knots with $n$ double points into classes. Given a singular knot with $n$ double points we define its configuration as the diagram which results after drawing a circle and marking $2 n$ points on it in the order that the group generators appear in (1.1). The points corresponding to generators with the same index are joined by dashed line. The configuration corresponding to the singular knot shown 


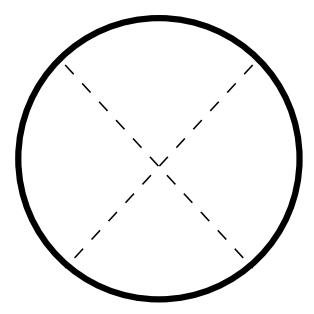

Figure 2: Configuration corresponding to the singular knot shown in fig. 1.

in fig. 1 has been depicted in fig. 2. Two singular knots are in the same class if they possess the same configuration. This assignment of configurations to singular knots, suggested by the operators (1.1), is the same as the standard one used in knot theory (see, for example, 21]).

Though the general form of the operators (1.1) might look somehow cumbersome, it is straightforward to write down the operator (1.1) for specific cases. Let us illustrate this with an example. Let us consider the singular knot depicted in fig. 1. One first assigns to each singular point the triple made by the two values that the parameter $v$ takes and a group generator with some group index. Then one chooses one of the double points as base point and travels along the knot building a product of Wilson lines and generators as they are encountered in the journey. For the oriented singular knot in fig. 1 the resulting operator is:

$$
(2 x)^{2} \operatorname{Tr}\left[T^{a_{1}} U\left(s_{1}, s_{2}\right) T^{a_{2}} U\left(s_{2}, t_{1}\right) T^{a_{1}} U\left(t_{1}, t_{2}\right) T^{a_{2}} U\left(t_{2}, s_{1}\right)\right] .
$$

As we will show in the following section the operators (1.1) are gauge invariant. Their form constitutes a field-theory-based simple proof of the theorem by Birman and Lin [21] which states that the coefficient of order $n$ of the expansion of a polynomial invariant (after replacing its variable by $e^{x}$ ) is a Vassiliev invariant of order $n$. Indeed, (1.1) shows that the perturbative expansion of a polynomial invariant of an oriented knot with $n$ double points starts at order $n$. All the coefficients of lower order have therefore vanished and thus all these coefficients are Vassiliev invariants of order lower than $n$.

The expression (1.1) constitutes also a field-theory-based simple proof of the theorem by Bar-Natan [24] which states that semi-simple Lie algebras are Vassiliev invariants for singular knots which can be integrated to Vassiliev invariants for non-singular knots. The zero-order of the perturbative expansion of the vacuum expectation value of the operators (1.1), which is 
just the result of setting $U=1$, shows that semi-simple Lie algebras lead to Vassiliev invariants of order $n$ for oriented singular knots with $n$ double points.

These two examples constitute a sign of the wide variety of applications that the operators (1.1) have. In this paper we will study the perturbative series expansion associated to the vacuum expectation value of (1.1) in the light-cone gauge. As a result we obtain a generalization of the Kontsevich integral for Vassiliev invariants [22, 23] which is valid in the case of singular knots. We will present also the general form of the Vassiliev invariant of order $n$ for a singular knot with $n-1$ double points. This last result allows to complete very simply the next to the top row of an actuality table.

The paper is organized as follows. In sect. 2 we present the construction of the gauge invariant operators associated to singular knots. In sect. 3 we discuss some immediate consequences which follow very simply from the form of these operators. In sect. 4 we analyze the new operators in the light-cone gauge and we present a generalization of the Kontsevich integral for ordinary knots to the case of singular knots. In sect. 5 we carry out one more application: we present the general form of the Vassiliev invariants of order $n$ for singular knots with $n-1$ singularities. Finally, in sect. 6 we state our conclusions. 


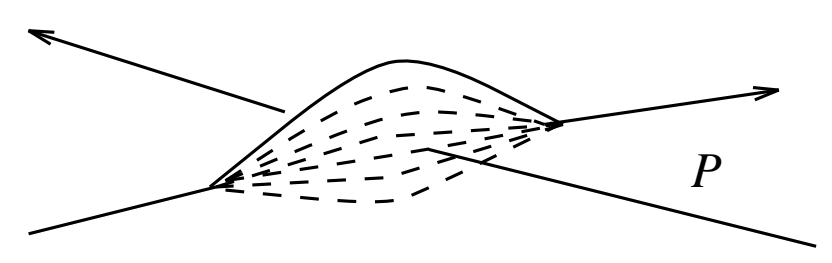

Figure 3: Resolution of a singular knot by a continuous of paths.

\section{Operators for singular knots}

In this section we construct the gauge invariant operators associated to singular knots in the context of Chern-Simons gauge theory. We will first review some standard properties of Wilson lines. Then, after the analysis of the variation of their vacuum expectation value, we will carry out the construction of the operators (1.1).

Let us consider Chern-Simons gauge theory on a boundaryless smooth three-manifold $M$ for a semi-simple gauge group $G$. The action of the theory, $S(A)$, is built out of the Chern-Simons three-form,

$$
S(A)=\frac{k}{4 \pi} \int_{M} \operatorname{Tr}\left(A \wedge d A+\frac{2}{3} A \wedge A \wedge A\right),
$$

where $A_{\mu}=A_{\mu}^{a} T^{a}$ is a $G$-connection, $k$ an integer parameter, and Tr denotes the trace in the fundamental representation of the gauge group $G$. The quantities $T^{a}, a=1, \ldots, \operatorname{dim} G$, are the group generators, which are normalized so that $\operatorname{Tr}\left(T^{a} T^{b}\right)=\frac{1}{2} \delta^{a b}$. One salient property of the action (2.1) is that its variation respect to the gauge connection has the form:

$$
\frac{\delta}{\delta A_{\mu}^{a}(x)} S(A)=\frac{k}{8 \pi} \epsilon^{\mu \nu \rho} F_{\nu \rho}^{a}(x),
$$

where $F_{\nu \rho}$ is the curvature of $A_{\mu}$.

The natural observables of Chern-Simons gauge theory are Wilson loops and graphs (or spin networks). Their vacuum expectation values are related to knot, link and graph invariants. In this work we are interested in analyzing the variation of the vacuum expectation values of Wilson loops under a deformation of their associated path. Actually, we need to study first a slightly more involved object: the Wilson line. Let us first recall its definition 


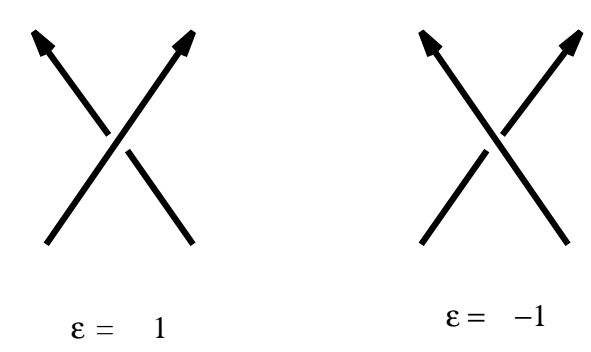

Figure 4: Signatures at the crossings.

and review some of its properties. Let $\gamma$ be a path in $M$, i.e. a smooth map $\gamma:[s, t] \rightarrow M$. The associated Wilson line $U_{\gamma}(s, t)$ is defined as:

$$
U_{\gamma}(s, t)=\mathrm{P} \exp \int_{s}^{t} d v \dot{\gamma}(v) A(\gamma(v)),
$$

where $\mathrm{P}$ denotes path ordering. In (2.3) there is an implicit choice of a representation of the gauge group. The vacuum expectation value of a Wilson line is defined as the functional integral:

$$
\left\langle U_{\gamma}(s, t)\right\rangle=\int[D A] U_{\gamma}(s, t) \mathrm{e}^{i S(A)} .
$$

We will consider a family of smooth paths $\gamma_{u}$ parametrized by the continuous parameter $u$, such that for $u=0$ the path $\gamma_{0}$ possesses a self-intersection at some point $P \in M$, i.e. for $u=0$ it has a double point. For $u>0(u<0)$ the path presents an overcrossing (undercrossing) near the point $P$. A family of paths with these features has been pictured in fig. 3. The path $\gamma_{0}(v)$ has a double point at $v=s_{1}$ and $v=t_{1}$ with $s_{1}<t_{1}$. Paths $\gamma_{u}(v)$ with $u \neq 0$ are different than $\gamma_{0}(v)$ only in a region in parameter space around $v=s_{1}$. The derivative of $\gamma_{u}(v)$ respect to $u$ is only non-zero in that region. It vanishes away from $v=s_{1}$, in particular at $v=t_{1}$. In the resolution of a double point we will call overcrossing (undercrossing) to the one which leads to a crossing with positive (negative) sign, as depicted in fig. 1 . Our goal is to study the first derivative of the vacuum expectation value of the Wilson line $U_{\gamma_{u}}(s, t)$ respect to the parameter $u$. As stated in the introduction, due to the topological character of Chern-Simons gauge theory, one expects a step-function behavior for $\left\langle U_{\gamma_{u}}(s, t)\right\rangle$ as a function of $u$ in a neighborhood of $u=0$. This implies the presence of a delta function in its derivative. As we show below, this is in fact what we find. 
Our starting point is the well known formula for the variation of a Wilson line under a deformation of its path:

$$
\frac{d}{d u} U_{\gamma_{u}}(s, t)=\int_{s}^{t} d v \gamma_{u}^{\prime \mu}(v) \dot{\gamma}_{u}^{\nu}(v) U_{\gamma_{u}}(s, v) F_{\mu \nu}(\gamma(v)) U_{\gamma_{u}}(v, t)
$$

where we have denoted derivatives respect to the path parameter by a dot, and derivatives respect to $u$ by a prime. Recall that $\gamma_{u}^{\prime}(v)$ is only different from zero in a region in parameter space around $v=s_{1}$. Another important property of the Wilson line which will be used later is its behavior under a functional derivation respect to the gauge connection:

$$
\frac{\delta}{\delta A_{\mu}^{a}(x)} U_{\gamma_{u}}(s, t)=\int_{s}^{t} d w \dot{\gamma}_{u}^{\mu}(w) \delta^{(3)}\left(x, \gamma_{u}(w)\right) U_{\gamma_{u}}(s, w) T^{a} U_{\gamma_{u}}(w, t) .
$$

Taking into account (2.5) and (2.2) and integrating by parts in connection space one can write the derivative respect to $u$ of the vacuum expectation value (2.4) as:

$$
\begin{gathered}
\frac{d}{d u}\left\langle U_{\gamma_{u}}(s, t)\right\rangle= \\
\frac{4 \pi i}{k} \int[D A] \mathrm{e}^{i S(A)} \int_{s}^{t} d v \epsilon_{\mu \nu \rho} \gamma_{u}^{\prime \mu}(v) \dot{\gamma}_{u}^{\nu}(v) \frac{\delta}{\delta A_{\rho}^{a}\left(\gamma_{u}(v)\right)} U_{\gamma_{u}}(s, v) T^{a} U_{\gamma_{u}}(v, t) .
\end{gathered}
$$

Using (2.6) this can be written as:

$$
\begin{aligned}
& \frac{d}{d u}\left\langle U_{\gamma_{u}}(s, t)\right\rangle=\frac{4 \pi i}{k} \int[D A] \mathrm{e}^{i S(A)} \int_{s}^{t} d v \epsilon_{\mu \nu \rho} \gamma_{u}^{\prime \mu}(v) \dot{\gamma}_{u}^{\nu}(v) \\
& \quad\left[\int_{s}^{v} d w \dot{\gamma}_{u}^{\rho}(w) \delta^{(3)}\left(\gamma_{u}(v), \gamma_{u}(w)\right) U_{\gamma_{u}}(s, w) T^{a} U_{\gamma_{u}}(w, v) T^{a} U_{\gamma_{u}}(v, t)\right. \\
& \left.\quad+\int_{v}^{t} d w \dot{\gamma}_{u}^{\rho}(w) \delta^{(3)}\left(\gamma_{u}(v), \gamma_{u}(w)\right) U_{\gamma_{u}}(s, v) T^{a} U_{\gamma_{u}}(v, w) T^{a} U_{\gamma_{u}}(w, t)\right] .
\end{aligned}
$$

Due to the presence of the delta function in this expression, contributions come from the solutions to the equation

$$
\gamma_{u}(v)=\gamma_{u}(w)
$$


This equation has two types of solutions. The first type is the one-dimensional set of solutions $v=w$ for each value of $u$. The second type is made out of the solutions for which $v \neq w$. Notice that if there are not self-intersections the set of second-type solutions is empty. Since (2.8) is only non-vanishing for $v$ in a neighborhood of $s_{1}$, we have only one second-type solution: $u=0$, $v=s_{1}$ and $w=t_{1}$. The contribution comes from the second $w$-integral in (2.8).

Contributions of the first type are related to framing. Recall that in Chern-Simons gauge theory one does not have topological invariants unless a framing is introduced. If there is not framing there is a contribution proportional to the Gauss self-linking number which is metric dependent [11]. As argued in [13], the variation of this contribution under a deformation of the path leads in fact to (2.8) for contributions of the first type. In the case one introduces a framing, contributions of the first type vanish because the paths entering the delta function (the path itself and its companion) never get values at coincident points in three-space for $v=w$. Thus, if one considers framed knots, contributions of the first type can be ignored. In the case of framed knots there is always a contribution of the second type if the path has a self-intersection. Generically it will occur for values of $u, v$ and $w$ slightly displaced from from the previously assigned values at the intersection point. In the limit of zero width one recovers the initial values. The contribution is framing independent and one can therefore continue the analysis of (2.8) without taking into account the framing and ignoring the contributions of first type. See [19 for a detailed analysis of the contributions to (2.8) of the first type.

For the family of paths under consideration there is only one contribution of the second type in the integral (2.8). This occurs at $u=0, v=s_{1}$ and $w=t_{1}$. In a neighborhood of this solution, the delta-function can be written as:

$$
\delta^{(3)}\left(\gamma_{u}(v), \gamma_{u}(w)\right)=\frac{1}{\left|\Delta\left(0, s_{1}, t_{1}\right)\right|} \delta(u) \delta\left(v-s_{1}\right) \delta\left(w-t_{1}\right)
$$

where,

$$
\Delta(u, v, w)=\epsilon_{\mu \nu \rho} \gamma_{u}^{\prime \mu}(v) \dot{\gamma}_{u}^{\nu}(v) \dot{\gamma}_{u}^{\rho}(w) .
$$

Plugging this expression into the derivative (2.8) one finds that, as expected, 
it is proportional to a delta function in the variable $u$ :

$$
\frac{d}{d u}\left\langle U_{\gamma_{u}}(s, t)\right\rangle=\frac{4 \pi i}{k} \delta(u) \int[D A] \mathrm{e}^{i S(A)} U_{\gamma_{u}}\left(s, s_{1}\right) T^{a} U_{\gamma_{u}}\left(s_{1}, t_{1}\right) T^{a} U_{\gamma_{u}}\left(t_{1}, t\right) .
$$

In obtaining this expression from (2.8) one has to take into account that for the selected values of $u, v$ and $w$ one has $\Delta\left(0, s_{1}, t_{1}\right)>0$. Integrating in the variable $u$ in a region which encloses $u=0$ one finds the difference between $\left\langle U(s, t)_{+}\right\rangle$, the Wilson line with no intersection and an overcrossing at $P$ and $\left\langle U(s, t)_{-}\right\rangle$, the Wilson line with no intersections and an undercrossing at $P$ :

$$
\begin{aligned}
& \left\langle U(s, t)_{+}\right\rangle-\left\langle U(s, t)_{-}\right\rangle=\int_{-\eta}^{\eta} d u \frac{d}{d u}\left\langle U_{\gamma_{u}}(s, t)\right\rangle \\
& =\frac{4 \pi i}{k} \int[D A] \mathrm{e}^{i S(A)} U_{\gamma_{0}}\left(s, s_{1}\right) T^{a} U_{\gamma_{0}}\left(s_{1}, t_{1}\right) T^{a} U_{\gamma_{0}}\left(t_{1}, t\right),
\end{aligned}
$$

where $\eta$ is some positive small real number.

From the previous expression one can read very simply the form of the operator associated to a Wilson line with a double point at a point $P \in M$ which corresponds to the values $v=s_{1}$ and $v=t_{1}$ of its path parameter. The rule is very simple: the original Wilson line is splited into three sections. The operator is made out of the ordered product of the corresponding three Wilson lines with insertions of group generators at each joint. The operator obtained in (2.13) is gauge covariant. Recall that under a gauge transformation,

$$
A_{\mu} \rightarrow \Lambda^{-1}\left(\partial_{\mu}+A_{\mu}\right) \Lambda
$$

a Wilson line $U_{\gamma}(s, t)$ transforms as,

$$
U_{\gamma}(s, t) \rightarrow \Lambda^{-1}(\gamma(s)) U_{\gamma}(s, t) \Lambda(\gamma(t)) .
$$

Thus, the operator inserted in (2.13) transforms as:

$$
\begin{aligned}
& U_{\gamma}\left(s, s_{1}\right) T^{a} U_{\gamma}\left(s_{1}, t_{1}\right) T^{a} U_{\gamma}\left(t_{1}, t\right) \rightarrow \\
& \quad \Lambda^{-1}(\gamma(s)) U_{\gamma}\left(s, s_{1}\right) \Gamma^{a}\left(\gamma\left(s_{1}\right)\right) U_{\gamma}\left(s_{1}, t_{1}\right) \Gamma^{a}\left(\gamma\left(t_{1}\right)\right) U_{\gamma}\left(t_{1}, t\right) \Lambda(\gamma(t)),
\end{aligned}
$$

where,

$$
\Gamma^{a}(x)=\Lambda^{-1}(x) T^{a} \Lambda(x)=\lambda^{a b}(x) T^{b} .
$$


In this last equation we have used the fact that the set of group generators is invariant under conjugation. The quantities $\lambda^{a b}$ entering (2.17) satisfy:

$$
\lambda^{a b}(x) \lambda^{a c}(x)=\delta^{b c} .
$$

Using this property and the fact that $\gamma\left(s_{1}\right)=\gamma\left(t_{1}\right)$ one finds that, indeed, the operators entering (2.13) are gauge covariant:

$$
\begin{aligned}
& U_{\gamma}\left(s, s_{1}\right) T^{a} U_{\gamma}\left(s_{1}, t_{1}\right) T^{a} U_{\gamma}\left(t_{1}, t\right) \rightarrow \\
& \quad \Lambda^{-1}(\gamma(s)) U_{\gamma}\left(s, s_{1}\right) T^{a} U_{\gamma}\left(s_{1}, t_{1}\right) T^{a} U_{\gamma}\left(t_{1}, t\right) \Lambda(\gamma(t)) .
\end{aligned}
$$

The result (2.13) can be used to obtain the operator associated to a singular knot with one double point. Let us denote by $\psi_{+}$the vacuum expectation value corresponding to a Wilson loop when the double point at $P \in M$ is solved by an overcrossing, and $\psi_{-}$when it is solved by an undercrossing. The difference $\psi_{+}-\psi_{-}$will be given by the trace of (2.13) for a closed path, i.e. a path $\gamma$ where its end points are identified. One easily finds:

$$
\psi_{+}-\psi_{-}=2 x\left\langle\operatorname{Tr}\left[T^{a} U_{\gamma}\left(s_{1}, t_{1}\right) T^{a} U_{\gamma}\left(t_{1}, s_{1}\right)\right]\right\rangle,
$$

where, again, the subindex in the path $\gamma$ has been suppressed. In this equation we have made use of (1.2). The gauge invariance of the operator entering this expression follows trivially from (2.19).

As a consistency check of this equation, let us compute its lowest order contribution for a singular knot $K^{1}$ with one double point. We will denote by $K_{+}$and $K_{-}$the non-singular knots which correspond to the resolution of the double point by an overcrossing and an undercrossing, respectively. If one chooses a framing in which the linking number of a knot and its companion knot coincide with the writhe (for some projection of the knot) one has that at the lowest non-trivial order the vacuum expectation value of its associated Wilson loop is proportional to the writhe. Indeed, one finds, for example, for $\psi_{+}$:

$$
\psi_{+}=\operatorname{dim} R\left(1+x C_{2}(R) \sum_{i} \epsilon_{i}\right),
$$

where the sum is over all the crossings $i$, and $\epsilon_{i}$ is the sign of the crossing $i$. In (2.21) $C_{2}(R)=\operatorname{Tr}\left(T^{a} T^{a}\right) / \operatorname{dim} R$ and $R$ is the representation carried by the knot. A similar equation holds for the lowest order of $\psi_{-}$. The difference 
$\psi_{+}-\psi_{-}$can be computed very simple because all the terms of their sums are the same for both knots except the one corresponding to the double point. The contribution from all but one, the double point, cancel. The contributions from the double point add and one ends with

$$
\psi_{+}-\psi_{-}=2 x \operatorname{dim} R C_{2}(R)=2 x \operatorname{Tr}\left(T^{a} T^{a}\right) .
$$

This is precisely what one obtains from (2.20) at lowest order.

Our next task is to analyze differences corresponding to two singular knots with one double point to obtain the form of the operators for singular knots with two double points. The strategy is the following. Let us consider a singular knot with two double points. One of them is located at a point $P_{1} \in M$ and the corresponding values of the knot parameter are $v=s_{1}$ and $v=t_{1}$, with $s_{1}<t_{1}$. The second one is located at $P_{2} \in M$ for $v=s_{2}$ and $v=t_{2}$, with $s_{2}<t_{2}$. The resolution of the second double point into an overcrossing and an undercrossing involves two singular knots with one double point whose invariants will be denoted by $\psi_{+}^{1}$ and $\psi_{-}^{1}$, respectively. The singular knot invariants associated to $\psi_{ \pm}^{1}$ can be written as the vacuum expectation values:

$$
\psi_{ \pm}^{1}=2 x\left\langle\operatorname{Tr}\left[T^{a} U_{\gamma_{ \pm}}\left(s_{1}, t_{1}\right) T^{a} U_{\gamma_{ \pm}}\left(t_{1}, s_{1}\right)\right]\right\rangle,
$$

where $\gamma_{ \pm}$denotes the paths associated to the singular knots corresponding to the two resolutions. In order to find out the operator corresponding to a singular knot with two double points we must consider a family of singular knots parametrized by $u$ such that for $u>0(u<0)$ the singular knot is equivalent under rigid vertex isotopy to $\psi_{+}^{1}\left(\psi_{-}^{1}\right)$, and for $u=0$ it possesses a second double point at $P_{2}$. Making use of this family, the difference $\psi_{+}^{1}-\psi_{-}^{1}$ can be written as:

$$
\psi_{+}^{1}-\psi_{-}^{1}=\int_{-\eta}^{\eta} d u \frac{d}{d u} \psi_{\gamma_{u}}^{1},
$$

where,

$$
\psi_{\gamma_{u}}^{1}=2 x\left\langle\operatorname{Tr}\left[T^{a} U_{\gamma_{u}}\left(s_{1}, t_{1}\right) T^{a} U_{\gamma_{u}}\left(t_{1}, s_{1}\right)\right]\right\rangle .
$$

To analyze the derivative of $\psi_{\gamma_{u}}^{1}$ respect to $u$ let us assume that $s_{1}<$ $s_{2}<t_{1}$. As it will become clear from the analysis, the other case, $t_{1}<s_{2}$, 


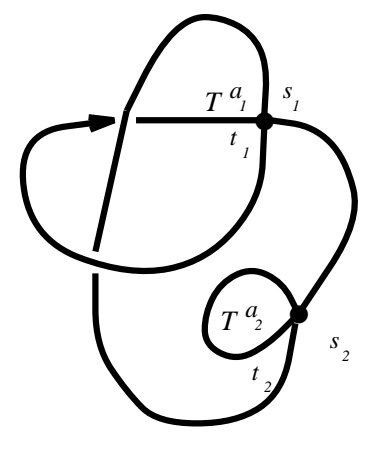

$a$

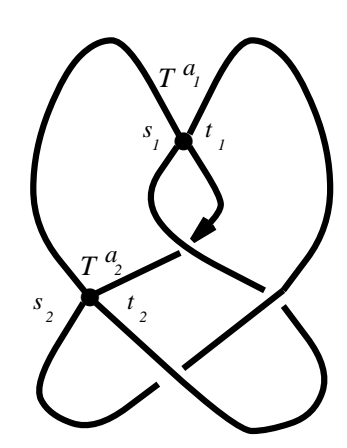

$b$

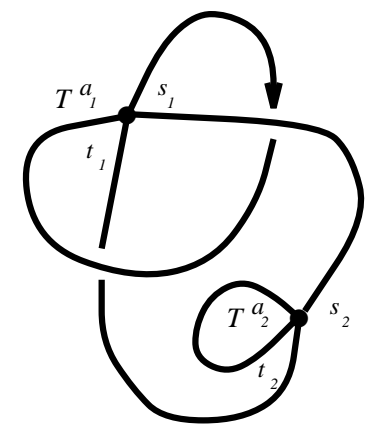

$c$

Figure 5: Examples of the three types of contributions.

can be treated similarly. Using (2.5) and (2.2) and integrating by parts in connection space one finds:

$$
\begin{gathered}
\quad \frac{d}{d u} \psi_{\gamma_{u}}^{1}=(2 x)^{2} \int[D A] \mathrm{e}^{i S(A)} \\
\int_{s}^{t} d v \epsilon_{\mu \nu \rho} \gamma_{u}^{\prime \mu}(v) \dot{\gamma}_{u}^{\nu}(v) \frac{\delta}{\delta A_{\rho}^{b}\left(\gamma_{u}(v)\right)} \operatorname{Tr}\left[T^{a} U_{\gamma_{u}}\left(s_{1}, v\right) T^{b} U_{\gamma_{u}}\left(v, t_{1}\right) T^{a} U_{\gamma_{u}}\left(t_{1}, s_{1}\right)\right]
\end{gathered}
$$

which, after using (2.6) can be written as:

$$
\begin{gathered}
\frac{d}{d u} \psi_{\gamma_{u}}^{1}=(2 x)^{2} \int[D A] \mathrm{e}^{(i S(A))} \int_{s}^{t} d v \epsilon_{\mu \nu \rho} \gamma_{u}^{\prime \mu}(v) \dot{\gamma}_{u}^{\nu}(v) \\
{\left[\int_{s_{1}}^{v} d w \dot{\gamma}_{u}(w) \delta^{(3)}\left(\gamma_{u}(v), \gamma_{u}(w)\right) \operatorname{Tr}\left[T^{a} U_{\gamma_{u}}\left(s_{1}, w\right) T^{b} U_{\gamma_{u}}(w, v) T^{b} U_{\gamma_{u}}\left(v, t_{1}\right) T^{a} U_{\gamma_{u}}\left(t_{1}, s_{1}\right)\right],\right.} \\
+\int_{v}^{t_{1}} d w \dot{\gamma}_{u}(w) \delta^{(3)}\left(\gamma_{u}(v), \gamma_{u}(w)\right) \operatorname{Tr}\left[T^{a} U_{\gamma_{u}}\left(s_{1}, v\right) T^{b} U_{\gamma_{u}}(v, w) T^{b} U_{\gamma_{u}}\left(w, t_{1}\right) T^{a} U_{\gamma_{u}}\left(t_{1}, s_{1}\right)\right], \\
\left.+\int_{t_{1}}^{s_{1}} d w \dot{\gamma}_{u}(w) \delta^{(3)}\left(\gamma_{u}(v), \gamma_{u}(w)\right) \operatorname{Tr}\left[T^{a} U_{\gamma_{u}}\left(s_{1}, v\right) T^{b} U_{\gamma_{u}}\left(v, t_{1}\right) T^{a} U_{\gamma_{u}}\left(t_{1}, w\right) T^{b} U_{\gamma_{u}}\left(w, s_{1}\right)\right]\right] .
\end{gathered}
$$

As in the previous case we must now study the solutions to equation (2.9). Certainly, there are solutions of the first type. These, as argued before, can 
be ignored. In the region where $\gamma_{u}^{\prime \nu}(v) \neq 0$ we have only one solution of the second type: $v=s_{2}$ and $w=t_{2}$. The only term of the three-dimensional delta function in (2.27) which contributes can be written as,

$$
\frac{1}{\left|\Delta\left(0, s_{2}, t_{2}\right)\right|} \delta(u) \delta\left(v-s_{2}\right) \delta\left(w-t_{2}\right)
$$

where $\Delta(u, v, w)$ is given in (2.11). Only one of the three integrals in (2.27) contribute. One must distinguish two cases: $t_{2}<t_{1}$ and $t_{2}>t_{1}$. In the first case one ends with (recall that we are considering the situation $s_{2}<t_{1}$ ):

$$
(2 x)^{2} \delta(u) \int[D A] \mathrm{e}^{i S(A)} \operatorname{Tr}\left[T^{a} U_{\gamma_{u}}\left(s_{1}, s_{2}\right) T^{b} U_{\gamma_{u}}\left(s_{2}, t_{2}\right) T^{b} U_{\gamma_{u}}\left(t_{2}, t_{1}\right) T^{a} U_{\gamma_{u}}\left(t_{1}, s_{1}\right)\right],
$$

while in the second case,

$$
(2 x)^{2} \delta(u) \int[D A] \mathrm{e}^{i S(A)} \operatorname{Tr}\left[T^{a} U_{\gamma_{u}}\left(s_{1}, s_{2}\right) T^{b} U_{\gamma_{u}}\left(s_{2}, t_{1}\right) T^{a} U_{\gamma_{u}}\left(t_{1}, t_{2}\right) T^{b} U_{\gamma_{u}}\left(t_{2}, s_{1}\right)\right] .
$$

It is also clear that in the situation $t_{1}<s_{2}$ we would have obtained:

$(2 x)^{2} \delta(u) \int[D A] \mathrm{e}^{i S(A)} \operatorname{Tr}\left[T^{a} U_{\gamma_{u}}\left(s_{1}, t_{1}\right) T^{a} U_{\gamma_{u}}\left(t_{1}, s_{2}\right) T^{b} U_{\gamma_{u}}\left(s_{2}, t_{2}\right) T^{b} U_{\gamma_{u}}\left(t_{2}, s_{1}\right)\right]$.

Using (2.24) we finally obtain the final expression for $\psi_{+}^{1}-\psi_{-}^{1}$. There are three cases depending on the values of $s_{2}$ and $t_{2}$ :

$$
\begin{aligned}
& (2 x)^{2}\left\langle\operatorname{Tr}\left[T^{a} U\left(s_{1}, s_{2}\right) T^{b} U\left(s_{2}, t_{2}\right) T^{b} U\left(t_{2}, t_{1}\right) T^{a} U\left(t_{1}, s_{1}\right)\right]\right\rangle, s_{1}<s_{2}<t_{2}<t_{1}, \\
& (2 x)^{2}\left\langle\operatorname{Tr}\left[T^{a} U\left(s_{1}, s_{2}\right) T^{b} U\left(s_{2}, t_{1}\right) T^{a} U\left(t_{1}, t_{2}\right) T^{b} U\left(t_{2}, s_{1}\right)\right]\right\rangle, s_{1}<s_{2}<t_{1}<t_{2}, \\
& (2 x)^{2}\left\langle\operatorname{Tr}\left[T^{a} U\left(s_{1}, t_{1}\right) T^{a} U\left(t_{1}, s_{2}\right) T^{b} U\left(s_{2}, t_{2}\right) T^{b} U\left(t_{2}, s_{1}\right)\right]\right\rangle, \quad s_{1}<t_{1}<s_{2}<t_{2},
\end{aligned}
$$

where we have suppressed the subindex of the Wilson line which denoted the path. The three different situations have been depicted in fig. 5a, 5b and 5c. Notice that the rule to write down the corresponding operators is very simple. One labels the double points, chooses one of them as base point, and 
travels along the singular knot inserting a generator at each double point, and a Wilson line for each section between double points.

The results (2.32) can written in a compact form introducing the triples $\tau_{i}=\left\{s_{i}, t_{i}, T^{a_{i}}\right\}$ for $i=1,2$ and the set $\left\{s_{1}, t_{1}, s_{2}, t_{2}\right\}$. Ordering the elements of this set into $\left\{w_{1}, w_{2}, w_{3}, w_{4}\right\}, w_{i}<w_{i+1}$, and constructing the map $\phi\left(w_{i}\right)$ which assigns to $w_{i}$ the index of the group generator in the triple to which $w_{i}$ belongs, one can write the three cases as:

$\psi_{+}^{1}-\psi_{-}^{1}=$

$(2 x)^{2}\left\langle\operatorname{Tr}\left[T^{\phi\left(w_{1}\right)} U\left(w_{1}, w_{2}\right) T^{\phi\left(w_{2}\right)} U\left(w_{2}, w_{3}\right) T^{\phi\left(w_{3}\right)} U\left(w_{3}, w_{4}\right) T^{\phi\left(w_{4}\right)} U\left(w_{4}, w_{1}\right)\right]\right\rangle$

where we have dropped the subindex $\gamma$ of $U$ which specified the path. This is precisely the operator (1.1) for $n=2$.

The picture that emerges for the general situation is the following. Given an operator for a singular knot with $n-1$ double points, the operator corresponding to a singular knot with one more double point will be the result of inserting an operator of the form $U T^{a} U$ at the two sections of the singular knot involved in the additional double point. Let us assume that we have a singular knot with $n$ double points and that we have constructed the operators for all singular knots with $n-1$ double points. The $n^{\text {th }}$ double point will generally involve two sections, the one corresponding to the open interval $\left(w_{i}, w_{i+1}\right)$ in parameter space, and the one corresponding to $\left(w_{j}, w_{j+1}\right)$, $w_{i}<w_{j}$. It might happen that the resolution of the $n^{\text {th }}$ double point involves only one section, as it is the case of the second double point shown in figs. 5a and 5c. The analysis that follows holds also for that case. The new double point is located at the points $s_{n}$ and $t_{n}, s_{n}<t_{n}$, in parameter space. Of course, $w_{i}<s_{n}<w_{i+1}$ and $w_{j}<t_{n}<w_{j+1}$. The operators associated to the singular knots with $n-1$ double points in the resolution of the new double point will have the form:

$$
(2 x)^{n-1} \operatorname{Tr}\left[\cdots U\left(w_{i}, w_{i+1}\right) \cdots U\left(w_{j}, w_{j+1}\right) \cdots\right] .
$$

We need to compute the difference of the vacuum expectation values associated to these operators, $\psi_{+}^{n-1}-\psi_{-}^{n-1}$, which, as before, will be computed studying the first derivative respect to $u$ of the vacuum expectation value of 
the members of a family parametrized to $u$ which continuously connects one resolution to the other and that for $u=0$ corresponds to the singular knot with $n$ double points. We have:

$$
\psi_{+}^{n-1}-\psi_{-}^{n-1}=\int_{-\eta}^{\eta} d u \frac{d}{d u} \psi_{\gamma_{u}}^{n-1},
$$

where

$$
\psi_{\gamma_{u}}^{n-1}=(2 x)^{n-1}\left\langle\operatorname{Tr}\left[\cdots U_{\gamma_{u}}\left(w_{i}, w_{i+1}\right) \cdots U_{\gamma_{u}}\left(w_{j}, w_{j+1}\right) \cdots\right]\right\rangle .
$$

As in the previous cases the loop $\gamma_{u}$ differs from $\gamma_{0}$ only in a region near the point $s_{n}$ in parameter space. Only in a small neighborhood of $s_{n}$ the derivative $\gamma_{u}^{\prime}$ does not vanish. To compute the derivative of (2.36) we must proceed as before. The use of (2.5) leads to a replacement of $U_{\gamma_{u}}\left(w_{i}, w_{i+1}\right)$ by $U_{\gamma_{u}}\left(w_{i}, v\right) T^{a_{n}} U_{\gamma_{u}}\left(v, w_{i+1}\right)$ :

$$
\begin{aligned}
& \frac{d}{d u} \psi_{\gamma_{u}}^{n-1}=(2 x)^{n} \int[D A] \mathrm{e}^{i S(A)} \int_{s}^{t} d v \epsilon_{\mu \nu \rho} \gamma_{u}^{\prime \mu}(v) \dot{\gamma}_{u}^{\nu}(v) \\
& \frac{\delta}{\delta A_{\rho}^{a_{n}}\left(\gamma_{u}(v)\right)} \operatorname{Tr}\left[\cdots U_{\gamma_{u}}\left(w_{i}, v\right) T^{a_{n}} U_{\gamma_{u}}\left(v, w_{i+1}\right) \cdots U_{\gamma_{u}}\left(w_{j}, w_{j+1}\right) \cdots\right] .
\end{aligned}
$$

The functional derivative respect to $A_{\rho}^{a_{n}}\left(\gamma_{u}(v)\right)$ acts on each of the Wilson lines present in (2.37). However, due to the presence of the delta function, upon computing the functional derivative (see (2.6)), the only contribution which survives is the one coming from the Wilson line $U_{\gamma_{u}}\left(w_{j}, w_{j+1}\right)$. One obtains in this way an insertion of $U_{\gamma_{u}}\left(w_{j}, w\right) T^{a_{n}} U_{\gamma_{u}}\left(w, w_{j+1}\right)$ in the place of $U_{\gamma_{u}}\left(w_{j}, w_{j+1}\right)$ and then, after integrating in $v$ and $w$, one finds:

$$
\begin{aligned}
\frac{d}{d u} \psi_{\gamma_{u}}^{1}=(2 x)^{n} \delta(u)\left\langle\operatorname { T r } \left[\cdots U_{\gamma_{u}}\left(w_{i}, s_{n}\right) T^{a_{n}} U_{\gamma_{u}}\left(s_{n}, w_{i+1}\right) \cdots\right.\right. \\
\left.\left.\cdots U_{\gamma_{u}}\left(w_{j}, t_{n}\right) T^{a_{n}} U_{\gamma_{u}}\left(t_{n}, w_{j+1}\right) \cdots\right]\right\rangle
\end{aligned}
$$

which, after using (2.35), leads to:

$\psi_{+}^{n-1}-\psi_{-}^{n-1}=$ 


$$
(2 x)^{n}\left\langle\operatorname{Tr}\left[\cdots U\left(w_{i}, s_{n}\right) T^{a_{n}} U\left(s_{n}, w_{i+1}\right) \cdots U\left(w_{j}, t_{n}\right) T^{a_{n}} U\left(t_{n}, w_{j+1}\right) \cdots\right]\right\rangle
$$

where we have suppressed the subindices of the Wilson lines which denoted the loop. It is clear from the analysis that if in the resolution of the double point there is only one section involved the result is

$$
\begin{aligned}
& \psi_{+}^{n-1}-\psi_{-}^{n-1}= \\
& \quad(2 x)^{n}\left\langle\operatorname{Tr}\left[\cdots U\left(w_{i}, s_{n}\right) T^{a_{n}} U\left(s_{n}, t_{n}\right) T^{a_{n}} \cdots U\left(t_{n}, w_{i+1}\right) \cdots\right]\right\rangle,
\end{aligned}
$$

Both situations fit with the expression announced in (1.1).

The gauge invariance of the operators (1.1) follows from (2.15), (2.17) and (2.18). Indeed, one has that under the gauge transformation (2.14) the operator (1.1) becomes,

$$
\begin{gathered}
(2 x)^{n} \operatorname{Tr}\left[\Gamma^{\phi\left(w_{1}\right)}\left(\gamma\left(w_{1}\right)\right) U_{\gamma}\left(w_{1}, w_{2}\right) \Gamma^{\phi\left(w_{2}\right)}\left(\gamma\left(w_{2}\right)\right) U_{\gamma}\left(w_{2}, w_{3}\right) \Gamma^{\phi\left(w_{3}\right)}\left(\gamma\left(w_{3}\right)\right) \cdots\right. \\
\left.\cdots U_{\gamma}\left(w_{2 n-1}, w_{2 n}\right) \Gamma^{\phi\left(w_{2 n}\right)}\left(\gamma\left(w_{2 n}\right)\right) U_{\gamma}\left(w_{2 n}, w_{1}\right)\right]
\end{gathered}
$$

where we have made use of (2.14) and (2.15). Taking into account (2.17) this expression can be written as:

$$
\begin{aligned}
& (2 x)^{n} \lambda^{\phi\left(w_{1}\right) b_{1}}\left(\gamma\left(w_{1}\right)\right) \cdots \lambda^{\left.\phi\left(w_{2 n}\right)\right) b_{2 n}}\left(\gamma\left(w_{2 n}\right)\right) \\
& \quad \operatorname{Tr}\left[T^{b_{1}} U_{\gamma}\left(w_{1}, w_{2}\right) T^{b_{2}} U_{\gamma}\left(w_{2}, w_{3}\right) T^{b_{3}} \cdots U_{\gamma}\left(w_{2 n-1}, w_{2 n}\right) T^{b_{2 n}} U_{\gamma}\left(w_{2 n}, w_{1}\right)\right] .
\end{aligned}
$$

The indices $\phi\left(w_{1}\right), \ldots, \phi\left(w_{2 n}\right)$ are paired and precisely when $\phi\left(w_{i}\right)=\phi\left(w_{j}\right)$ one has $\gamma\left(w_{i}\right)=\gamma\left(w_{j}\right)$. We can then apply (2.18) $n$ times, obtaining (1.1) and therefore proving its gauge invariance.

In this section, we have shown the following:

Let $K^{n}$ be a singular knot with $n$ double points, and let us assign to each double point $i$ a triple $\tau_{i}=\left\{s_{i}, t_{i}, T^{a_{i}}\right\}$ where $s_{i}$ and $t_{i}, s_{i}<t_{i}$, are the values 
of the $K^{n}$-parameter at the double point, and $T^{a_{i}}$ is a group generator. The gauge invariant operator associated to the singular knot $K^{n}$ is:

$$
\begin{gathered}
(2 x)^{n} \operatorname{Tr}\left[T^{\phi\left(w_{1}\right)} U\left(w_{1}, w_{2}\right) T^{\phi\left(w_{2}\right)} U\left(w_{2}, w_{3}\right) T^{\phi\left(w_{3}\right)} \cdots\right. \\
\left.\cdots U\left(w_{2 n-1}, w_{2 n}\right) T^{\phi\left(w_{2 n}\right)} U\left(w_{2 n}, w_{1}\right)\right],
\end{gathered}
$$

where $\left\{w_{i} ; i=1, \ldots, 2 n\right\}, w_{i}<w_{i+1}$, is the set which results of ordering the values $s_{i}$ and $t_{i}$, for $i=1, \ldots, n$, and $\phi$ is a map which assigns to each $w_{i}$ the group generator in the triple to which it belongs. 


\section{$3 \quad$ Immediate applications}

In this section we will describe some immediate applications of the operators (1.1). First we will show that the theorem by Birman and Lin [21] proving that the $n$-order coefficient of the expansion of a (quantum group or Chern-Simons) polynomial knot invariant (after replacing its variable by $\mathrm{e}^{x}$ ) is a Vassiliev invariant of order $n$ easily follows from the form of the operators (1.1). Then, we will show that the form of the top row of actuality tables based on semi-simple Lie algebras provided by Bar-Natan [24] (Lie-algebra based weight systems) is also a direct consequence of the form of these operators. Actually, we will argue that our construction constitutes a gauge independent proof of the fact that Bar-Natan's weight systems can be integrated to Vassiliev invariants for non-singular knots.

\subsection{Birman and Lin theorem}

Some years ago Birman and Lin proved that if in any polynomial knot invariant with variable $t$ one substitutes $t \rightarrow \mathrm{e}^{x}$, and expand in powers of $x$, the coefficient of the power $x^{n}$ is a Vassiliev invariant of order $n$. A Vassiliev invariant of order $n$ vanishes for all singular knots with $n+1$ crossings. In the language of Chern-Simons gauge theory the theorem by Birman and Lin can be rephrased simply stating that the $n$-order term of the perturbative series expansion of the vacuum expectation value of a the Wilson loop associated to a given knot is a Vassiliev invariant of order $n$. We will now prove that this is so with the help of the operators (1.1).

The vacuum expectation value of the operators (1.1) provide an invariant for a singular knot with $n$ double points. This singular-knot invariant can be expressed as a signed sum of $2^{n}$ invariants for non-singular knots. These $2^{n}$ invariants are the perturbative series expansion of the vacuum expectation value of the corresponding Wilson loops. To show that the coefficient of order $n$ of these vacuum expectation values is a Vassiliev invariant or order $n$ is equivalent to prove that all the terms of order less than $n$ vanish in the

signed sum. But the signed sum is precisely the vacuum expectation value of the operator (1.1). Since the perturbative series expansion of these operators starts at order $n$, the proof of the theorem by Birman and Lin follows. 


\subsection{Bar-Natan's weight systems}

In [24] weight systems were introduced as elements of the top row of actuality tables for Vassiliev invariants. Using the Knotsevich integral, it was proved there that weight systems can be integrated to construct Vassiliev invariants. In this subsection we show that the operators (1.1) at lowest order are weight systems for the case of semi-simple Lie algebras. As we will argue below, our construction constitutes a gauge independent proof of the integrability of these weight systems to Vassiliev invariants for non-singular knots. We call it gauge independent because from the point of view of Chern-Simons gauge theory the original proof in [24] can be regarded as performed in a given gauge, the light-cone gauge. As it was shown in [30, 17], the Kontsevich integral appears in the context of Chern-Simons gauge theory when the theory is analyzed from a perturbative point of view in the light-cone gauge. In the analysis presented in this paper we have not been forced to make a gauge choice and therefore it is gauge independent.

Let us consider the vacuum expectation values of the operators (1.1) at order zero. Their expression is simply obtained setting $U=1$ in all the Wilson lines. Let us consider a singular knot $K^{n}$ with $n$ double points, $s_{i}, t_{i}$, with $s_{i}<t_{i}$, for $i=1, \ldots, n$, in parameter space. As usual, with these data we construct the triples: $\tau_{i}=\left\{s_{i}, t_{i}, T^{a_{i}}\right\}$. At lowest order in perturbation theory the operators (1.1) become the group factors,

$$
v_{n}\left(K^{n}\right)=(2 x)^{n} \operatorname{Tr}\left[T^{\phi\left(w_{1}\right)} T^{\phi\left(w_{2}\right)} T^{\phi\left(w_{3}\right)} \cdots T^{\phi\left(w_{2 n}\right)}\right],
$$

where the set $\left\{w_{1}, w_{2}, \ldots, w_{n}\right\}$, with $w_{j}<w_{j+1}$, is obtained by ordering the set $\left\{s_{i}, t_{i} ; i=1, \ldots, n\right\}$, and $\phi$ is the induced map which assigns to each $w_{j}$ the index of the group generator in the triple to which $w_{j}$ belongs. The indices entering (3.1) are paired. This allows to associate to each operator (3.1) a Feynman-like diagram in which the $2 n$ points are distributed in a circle and the ones which possess the same value of $\phi$ are joined by a line. In other words one can use simply the group-theoretical Feynman rules emerging from Chern-Simons gauge theory (see, for example, [17]). The resulting diagrams are the configuration diagrams for singular knots (as the one shown in fig. 2) and correspond to Bar-Natan's chord diagrams with $n$ chords [24].

It was proved in 24] that if weight systems are regarded as Vassiliev invariants for singular knots they can be integrated to Vassiliev invariants for non-singular knots. Let us consider an element of a weight system with 
$n$ chords, $B^{n}$, and any of its associated singular knots with $n$ double points, $K^{n}$. What was proved in 24] is that $B^{n}$ can be written as a signed sum of the $2^{n}$ Vassiliev invariants of non-singular knots which are generated when one performs the resolutions of the double points of $K^{n}$. The proof was done making use of the Kontsevich integral for Vassiliev invariants of non-singular knots. It was shown that the associated signed sum of Kontsevich integrals starts with a chord diagram of order $n$. But this is what has been done in this paper. Here, starting with the vacuum expectation values of Wilson loops associated to non-singular knots we have proved that the signed sum entering the invariant associated to the singular knot $K^{n}$ starts at order $n$, with the group factor (3.1). This group factor plays the role of the order- $n$ chord diagram appearing in Bar-Natan's approach.

As argued above, our work contains a gauge independent proof of BarNatan's theorem for the case of weight systems coming from semi-simple Lie algebras. Being a gauge theory, Chern-Simons gauge theory possesses a variety of pictures depending on the gauge in which it is studied. It was shown in [17] that in the light-cone gauge the picture which appears involves the Knotsevich integral. Thus, in a field-theory language, Bar-Natan's theorem was proved in the light-cone gauge. What is contained in this paper is a gauge independent proof of that theorem. Of course, the theorem has to hold in other gauges and in each gauge it can teach us something new. For example, it would be interesting to study it in a covariant gauge.

The group factors (3.1) are Vassiliev invariants of order $n$ for singular knots with $n$ double points. Of course, not all them are independent. Due to the Lie-algebra relations satisfied by the group generators there are linear relations between them. One important open problem in the theory of Vassiliev invariants is how many independent group factors (3.1) are at each order. The answer to this question is only known for low values of $n$ [24]. Once a choice of independent group factors is made one can build the top row of the actuality table for Vassiliev invariants of order $n$. 


\section{Kontsevich integral for singular knots}

In this section we will analyze the perturbative series expansion corresponding to the vacuum expectation value of the operators (1.1). We will carry out the analysis in the light-cone gauge. As a result, we will obtain a generalization of the Kontsevich integral for Vassiliev invariants which is also valid in the case of singular knots.

Chern-Simons gauge theory in the light-cone gauge was first studied in [四] where the vacuum expectation value of Wilson loops were analyzed from a non-perturbative point of view. In that paper a close relation between the vacuum expectation value of a Wilson line and the Knizhnik-Zamolodchikov [25] equations was pointed out. The loop structure of Chern-Simons gauge theory in the light-cone gauge has been studied in [26, 27]. Recently, the analysis of the perturbative series expansion of the vacuum expectation values of Wilson lines has been carried out [17], showing that it contains the Kontsevich integral for Vassiliev invariants of framed knots. In this section we will extend that analysis to the case in which the vacuum expectation values of the operators (1.1) associated to singular knots are considered.

To achieve our goal we will first review some basic facts about the lightcone gauge. We refer the reader to [1, 17] for more details. The light-cone gauge condition has the form:

$$
n^{\mu} A_{\mu}=0
$$

where $n^{\mu}$ is the light-like vector $(0,1,-1)$. Introducing the light-cone coordinates,

$$
x^{+}=x^{1}+x^{2}, \quad x^{-}=x^{1}-x^{2},
$$

and the corresponding light-cone components for the connection,

$$
A_{+}=A_{1}+A_{2}, \quad A_{-}=A_{1}-A_{2},
$$

the gauge-fixing condition (4.1) takes the form $A_{-}=0$. The Fadeev-Popov

ghost fields corresponding to the gauge fixing (4.1) decouple in the quantum action. The relevant part of this action for our purposes takes the form:

$$
S_{q}(A)=\frac{k}{4 \pi} \int_{M} d^{3} x\left(A_{+}^{a} \partial_{-} A_{0}^{a}-A_{0}^{a} \partial_{-} A_{+}^{a}\right) .
$$

In this section we will consider the case $M=\mathbf{R}^{3}$. 
As explained in [17], to consider (4.4) as the relevant quantum action to compute vacuum expectation values of gauge invariant operators is an oversimplification. The perturbation-theory formulation of gauge theories in non-covariant gauges is a very delicate issue [28]. On the one hand, propagators contain unphysical poles which have to be handled introducing some prescription; on the other hand, there is a residual gauge invariance. In the light-cone gauge the gauge fixing condition (4.1) is preserved under gauge transformations (2.14) such that $\Lambda$ depends only on $x^{0}$ and $x^{+}$. Both problems are related. It is a widespread belief that an adequate choice of prescription to avoid the unphysical poles solves the problem of the residual gauge invariance. In [4, 26, 17] the light-cone gauge has been analyzed using the Mandelstan-Leibbrandt prescription. We think that this prescription is not enough to solve the problem of the residual gauge invariance. As discussed in [17], the action (4.4) together with the Mandelstan-Leibbrandt prescription to circumvent the unphysical poles does not lead to a topological invariant expression for the vacuum expectation value of a Wilson loop. One needs to introduce a correction to the perturbative result to obtain the correct answer. We believe that this correction will be obtained after solving the problem related to the residual gauge invariance. The issue of the residual gauge invariance has been treated in [13] for the temporal gauge. For the purposes of this paper it is enough to consider (4.4) as the relevant quantum action of the theory. Of course, doing this we do not expect to obtain the correction term. However, we will certainly construct the generalization of the Kontsevich integral for the case of singular knots.

Another important issue which was discussed in [17] is the contribution from higher loops. This problem has been addressed in [26] in the case of the light-cone gauge, showing that it leads to an effect similar to the one observed in covariant gauges [31, 32, 33. As in [17, we will assume that, at most, higher loops account for a shift in the constant $k$.

The action (4.4) have two important properties: it does not have derivatives in the directions transverse to $x^{0}$, and it is quadratic in the fields. This last property implies that vacuum expectation values can be expressed in terms of the two-point correlation function. This constitutes an important simplification of the analysis of the perturbative series expansion associated to the vacuum expectation value of the operators (1.1).

As pointed out in [4, 17], in studying Chern-Simons gauge theory in the light-cone it is convenient to Wick rotate the theory into Euclidean space 


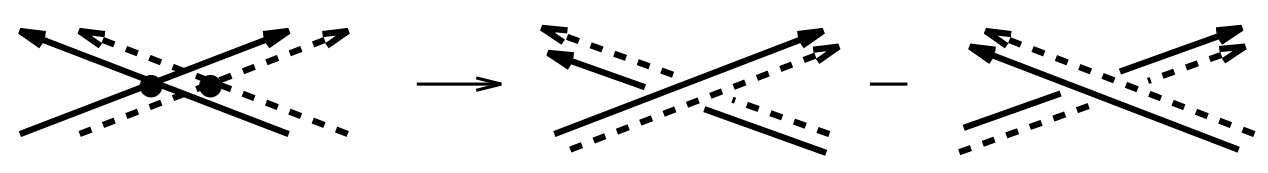

Figure 6: Resolution of a framed singular knot.

$\mathbf{R} \times \mathbf{C}$. If one denotes a point in Euclidean space by $(t, z)$, where $z=x^{1}+i x^{2}$, after introducing $A_{z}=A_{1}+i A_{2}$ and $A_{\bar{z}}=A_{1}-i A_{2}$ one finds [4, [17]:

$$
\begin{aligned}
\left\langle A_{\bar{z}}^{a}(x) A_{m}^{b}\left(x^{\prime}\right)\right\rangle & =0, \\
\left\langle A_{m}^{a}(x) A_{n}^{b}\left(x^{\prime}\right)\right\rangle & =\frac{4 \pi}{k} \delta^{a b} \epsilon_{m n} \frac{1}{2 \pi i} \frac{\delta\left(t-t^{\prime}\right)}{z-z^{\prime}},
\end{aligned}
$$

with $m, n=\{0, z\}$, and $\epsilon_{m n}$ is antisymmetric with $\epsilon_{0 z}=1$. The Feynman rules of the theory are given by the form of this propagator and the vertex associated to the Wilson lines:

$$
T^{a} \int d x^{\mu},
$$

where $T^{a}$ is a group generator and the integral is along the path corresponding to one of the Wilson lines entering (11.1).

The perturbative series expansion emerging out of the application of the Feynman rules corresponding to (4.5) and (4.6) in the computation of vacuum expectation values of Wilson loops was extensively analyzed in [17]. First, it was observed that due to the form of the propagator (4.5) one must consider Morse knots. Similarly, in the case under consideration, we will consider Morse singular knots where no double points appear at the extrema. In addition, a framing was introduced to deal with the problems generated by the singularities inherent to the propagator (4.5) when its end points coincide. For the case of singular knots with no double points at their extrema we can proceed in the same way.

We define framed singular knots considering the natural extension of framed knots. In a framed singular knot with $n$ double points the knot itself and its companion have $n$ self-intersections each. There is no intersection between the knot and its companion. The resolution of a double point of a framed singular knot is depicted in fig. 6. For reasons that will become clear below, it is convenient to locate the double points of the companion singular knot at the same heights as the double points of the singular knot itself. 
Framed singular knots induce a regularization which is achieved by replacing a propagator (4.5) joining two lines by a propagator which has one of its ends attached to the singular knot itself and the other to its companion. We must first prove that this regularization renders the terms of the perturbative series expansion finite after the zero-width limit is taken. But first we must recall some of the notation introduced in [17], and reformulate it to suit the case of singular knots.

Let us assume that the Morse singular knot $K^{n}$ possesses $2 m$ extrema and $n$ double points. In [17], a set of curves joining the different maxima and minima of $K^{n}$ where introduced. In the case of singular knots we need to enlarge this set. If one of the curves joining two extrema goes through a double point we will split it into two curves. We will consider therefore $2(m+n)$ curves $k^{i}, i=1, \ldots, 2(m+n)$, joining either two extrema, an extremum and a double point, or two double points. For each curve $k^{i}$ there is a one-to-one correspondence among the points of $k^{i}$ and the values that the variable $t$ takes. As in the non-singular case, $K^{n}$ can be regarded as a complex multivalued function of the variable $t$ with $2(m+n)$ components. This observation implies that there exist an alternative parametrization of $K^{n}: z_{i}(t), t \in I^{i}, i=1, \ldots, 2(m+n)$ where $z_{i}$ are the values that the variable $z$ takes on $k^{i}$ and $I=\left[t_{i}^{-}, t_{i}^{+}\right]$is the segment of $\mathbf{R}$ whose end-points are the values that the coordinate $t$ takes at the two extrema or double points joined by the curve $k^{i}$. With these data, a propagator attached to the curves $k^{i}$ and $k^{j}$ can be expressed as [17]:

$$
d x^{\mu} d x^{\nu}\left\langle A_{\mu}^{a}(x) A_{\nu}^{b}\left(x^{\prime}\right)\right\rangle \rightarrow \frac{4 \pi}{k} d t_{i} d t_{j} \frac{1}{2 \pi i} \delta\left(t_{i}-t_{j}\right) p_{i j} \frac{\dot{z}_{i}\left(t_{i}\right)-\dot{z}_{j}\left(t_{j}\right)}{z_{i}\left(t_{i}\right)-z_{j}\left(t_{j}\right)},
$$

where:

$$
p_{i j}= \begin{cases}1 & \text { if } k_{i} \text { and } k_{j} \text { have the same orientation, } \\ -1 & \text { if } k_{i} \text { and } k_{j} \text { have opposite orientations. }\end{cases}
$$

An analogous set of data, in which there are $2(m+n)$ curves $k_{\epsilon}^{i}, i=$ $1, \ldots, 2(m+n)$ and $z$ is replaced by $z^{\prime}$, is introduced for the companion knot. After performing one of the $t$-integrations, the regularization introduced by the framing leads to the following formulae for the propagator (4.7). For $i=j$ :

$$
\frac{4 \pi}{k} \frac{1}{2 \pi i} \frac{1}{2} d s \frac{\dot{z}_{i}(s)-\dot{z}_{i}^{\prime}(s)}{z_{i}(s)-z_{i}^{\prime}(s)}
$$




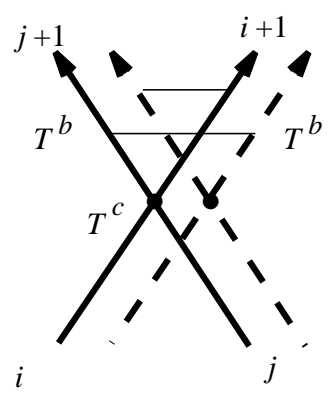

$a$

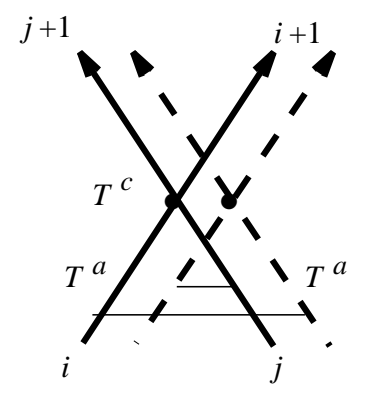

$b$

Figure 7: Propagator near a double point.

while for $i \neq j$ :

$$
\frac{4 \pi}{k} \frac{1}{2 \pi i} \frac{1}{2} d s\left(\frac{\dot{z}_{i}(s)-\dot{z}_{j}^{\prime}(s)}{z_{i}(s)-z_{j}^{\prime}(s)}+\frac{\dot{z}_{i}^{\prime}(s)-\dot{z}_{j}(s)}{z_{i}^{\prime}(s)-z_{j}(s)}\right) p_{i j} .
$$

It was shown in [17 that the regularization associated to framing renders the perturbative series expansion finite in the zero-width limit $(\epsilon \rightarrow 0)$ when the vacuum expectation value of a Wilson loop is considered. The novelty when performing the same analysis for the vacuum expectation values of the operators (1.1) is that now there are new divergences at the double points. However, the regularization achieved by the introduction of a framing makes the sum finite in the zero-width limit $(\epsilon \rightarrow 0)$. The only problematic contributions are those in which the two end-points of the propagators are attached to the two lines involved in the double point. There is always a contribution which approaches the double point from below and another from above. These contributions have been pictured in fig. 7 (the situation in which one of the directions is inverted can be treated similarly). Only the part of the singular knot near the double point has been depicted. First, notice that the group factors corresponding to both situations are the same. Indeed, for diagram $a$ one has a group factor,

$$
\operatorname{Tr}\left(T^{c} T^{b} X T^{c} T^{b} Y\right)
$$

while for diagram $b$ :

$$
\operatorname{Tr}\left(T^{c} X T^{a} T^{c} Y T^{a}\right)
$$


where $X$ and $Y$ are some combinations of group generators which are encountered as one travels along the singular knot. Certainly, (4.11) and (4.12) are the same group factor. The group factors (4.11) and (4.12) multiply an integrand which contains a product of propagator contributions. The relevant factor near the double point is given by the propagators shown in fig. 7 . If the double point takes place at height $t^{*}$, near the double point we must deal with integrals of the form:

$$
\begin{aligned}
& \int_{t^{*}}^{t_{>}} d s\left(\frac{\dot{z}_{i+1}(s)-\dot{z}_{j+1}^{\prime}(s)}{z_{i+1}(s)-z_{j+1}^{\prime}(s)}+\frac{\dot{z}_{i+1}^{\prime}(s)-\dot{z}_{j+1}(s)}{z_{i+1}^{\prime}(s)-z_{j+1}(s)}\right) p_{i+1, j+1}, \\
& \int_{t_{<}}^{t^{*}} d s\left(\frac{\dot{z}_{i}(s)-\dot{z}_{j}^{\prime}(s)}{z_{i}(s)-z_{j}^{\prime}(s)}+\frac{\dot{z}_{i}^{\prime}(s)-\dot{z}_{j}(s)}{z_{i}^{\prime}(s)-z_{j}(s)}\right) p_{i, j},
\end{aligned}
$$

where $t_{>}$and $t_{<}$are heights closed to $t^{*}$ such that $t_{>}>t^{*}$ and $t_{<}<t^{*}$. Of course, the integrals in (4.13) are the only part of the whole integral in which the contribution from the diagrams in fig. 7 differ. In the situation depicted in fig. 7, both $p_{i+1, j+1}=1$ and $p_{i, j}=1$ have value 1 . If one of the directions were inverted one would find $p_{i+1, j+1}=-1$ and $p_{i, j}=-1$. The important fact is that in all cases $p_{i+1, j+1}=p_{i, j}$. The problematic contributions from these integrals come from the region $s \rightarrow t^{*}$. Carrying out the integration one finds:

$$
\begin{aligned}
& \log \left(\frac{\left(z_{i+1}\left(t_{>}\right)-z_{j+1}^{\prime}\left(t_{>}\right)\right)\left(z_{i+1}^{\prime}\left(t_{>}\right)-z_{j+1}\left(t_{>}\right)\right)}{\left(z_{i+1}\left(t^{*}\right)-z_{j+1}^{\prime}\left(t^{*}\right)\right)\left(z_{i+1}^{\prime}\left(t^{*}\right)-z_{j+1}\left(t^{*}\right)\right)}\right) p_{i+1, j+1}, \\
& \log \left(\frac{\left(z_{i}\left(t^{*}\right)-z_{j}^{\prime}\left(t^{*}\right)\right)\left(z_{i}^{\prime}\left(t^{*}\right)-z_{j}\left(t^{*}\right)\right)}{\left(z_{i}\left(t_{<}\right)-z_{j}^{\prime}\left(t_{<}\right)\right)\left(z_{i}^{\prime}\left(t_{<}\right)-z_{j}\left(t_{<}\right)\right)}\right) p_{i, j} .
\end{aligned}
$$

The denominator of the argument of the first logarithm as well as the numerator of the argument of the second vanish in the zero-width limit, $\epsilon \rightarrow 0$, originating a logarithmic divergence. However, for a finite value of $\epsilon$ these contributions cancel with each other and one finds a finite answer for the sum in the zero-width limit. Notice that this cancellation occurs if the height of the double point of the companion singular knot is the same as the height of the singular knot itself. Situations with a ladder of propagators approaching the double point can be treated similarly. An equivalent picture that emerges from this description is that at the double point one must use a principal value prescription to avoid the singularity. We will describe this in more detail below when dealing with an explicit example. 


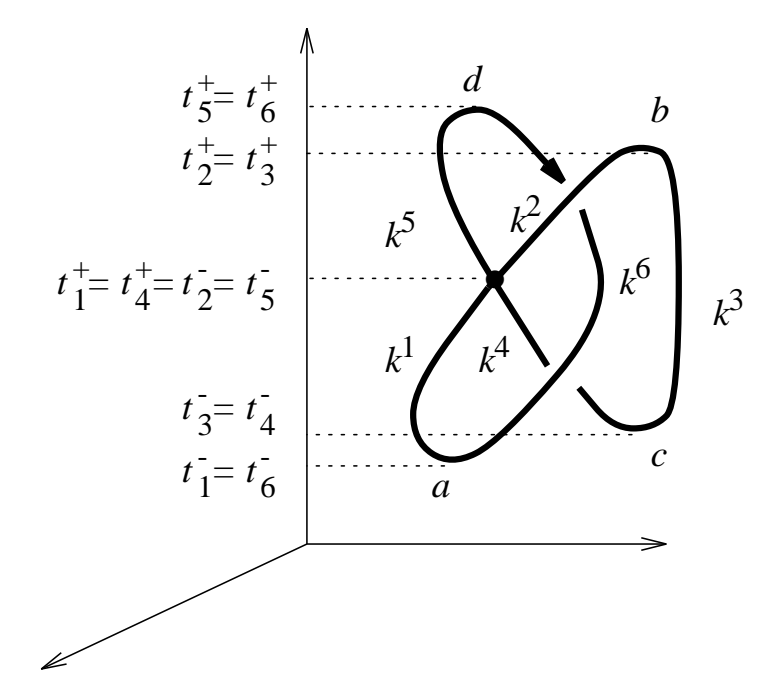

Figure 8: Example of a singular knot with a double point.

We are now in the position to write the general form of the terms of the perturbative series expansion. As in the case of non-singular knots, the delta function in the height present in the propagator (4.5) implies that the only non-vanishing propagators are those in which their two end-points have the same height. This observation allows to rearrange the contributions to the perturbative series expansion in the following way. Consider all possible pairings $\left\{z_{i}(s), z_{j}^{\prime}(s)\right\}$ of curves $k^{i}$ and $k_{\epsilon}^{j}, i, j=1, \ldots, 2(m+n)$, where $2 m$ is the number of extrema and $n$ is the number of double points. A contribution to the vacuum expectation value of an operator of the form (1.1), at order $q$ in perturbation theory, involves a path-ordered integral in the heights $s_{1}<\ldots<s_{r}<\ldots<s_{q}$, of a product of $q$ propagators (4.9) and (4.10) which contain,

$$
\prod_{r=1}^{q} \frac{d z_{i_{r}}\left(s_{r}\right)-d z_{j_{r}}^{\prime}\left(s_{r}\right)}{z_{i_{r}}\left(s_{r}\right)-z_{j_{r}}^{\prime}\left(s_{r}\right)} .
$$

Products of this type are characterized by $q$ pairs of numbers $\left(i_{r}, j_{r}\right)$ with $r=1, \ldots, q$. We will call the ordered set of these $q$ pairs an ordered pairing and we will denote it by $P_{q}$. One must take into account all the ordered pairings at each order in perturbation theory. This means that one must sum over all the $P_{q}$. The group factor associated to each ordered pairing $P_{q}$ is easily obtained by first placing group-generators at the two ends of 


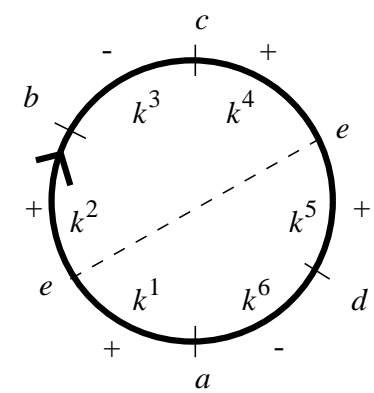

Figure 9: Representation of a singular knot with a double point on a circle.

the propagators involved in the ordered pairing, and then traveling along the kont building the resulting trace of generators. Of course, when doing this in the case of singular knots one encounters generators at the double points. We will denote the resulting group factor by $R\left(P_{q}\right)$. As in the case of non-singular knots we take care of all the signs originated from the $p_{i j}$ in the propagator (4.10) introducing

$$
s\left(P_{q}\right)=\prod_{r=1}^{q} p_{i_{r} j_{r}} .
$$

The full expression for the contribution to the perturbative series expansion at order $q$ takes the form:

$$
(2 x)^{n+q}\left(\frac{1}{2 \pi i}\right)^{q} \frac{1}{2^{q}} \sum_{P_{q}} \int_{\left.t_{P_{q}}^{-}<t_{1}<\ldots<t_{r}<\ldots<t_{m}<t_{P_{q}}^{+}\right)} \prod_{r=1}^{q} \frac{d z_{i_{r}}\left(t_{r}\right)-d z_{j_{r}}^{\prime}\left(t_{r}\right)}{z_{i_{r}}\left(t_{r}\right)-z_{j_{r}}^{\prime}\left(t_{r}\right)} R\left(P_{q}\right),
$$

where $t_{P_{q}}^{+}$and $t_{P_{q}}^{-}$are highest and lowest heights which can be reached by the last and first propagators of a given ordered pairing $P_{q}$. This expression has a close resemblance to the one presented in [17 for the case of nonsingular knots. There are, however, three important differences. First, the contributions in (4.17) begin at order $n$. Second, in the path integrals in (4.17) the double points are regarded as sources of two extrema, a maximum and a minimum. Third, the group factors $R\left(P_{q}\right)$ contain the group generators placed at the double points.

In order to illustrate the content of the integral (4.17) we will write down its explicit form for the singular knot depicted in fig. 8 at order $q=1$. Let us 


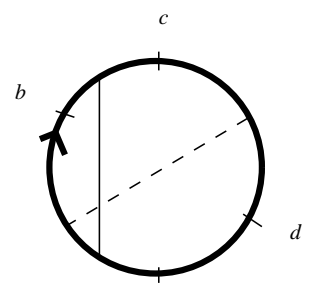

a

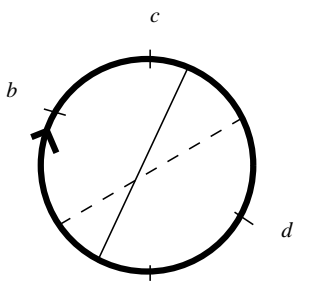

a

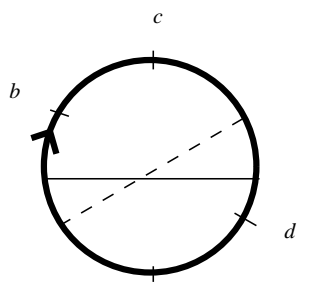

a

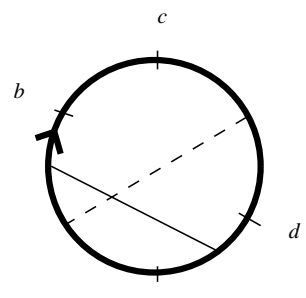

$a$

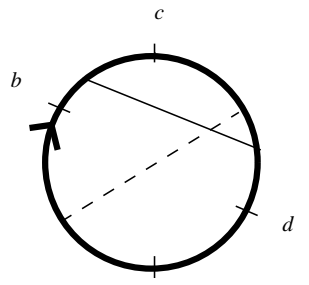

$a$
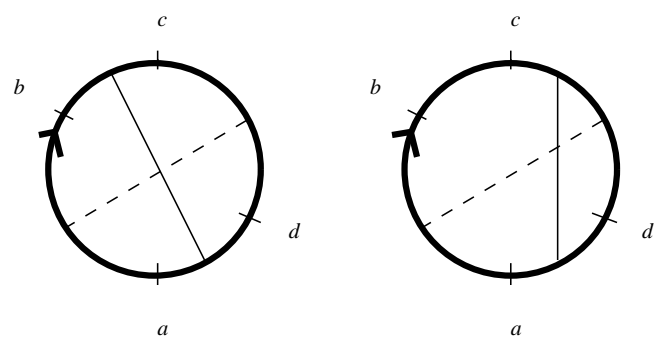

Figure 10: Contributions from the analysis on the associated circle.

denote that singular knot by $K^{1}$ and let us write down all the contributions to one of the group factors present for $q=1$. In order to analyze the contributions we will proceed as in [17]. As shown in fig. 8, we must consider six curves, $k^{1}, \ldots, k^{6}$, whose end-points are extrema, $a, b, c$ and $d$, and double points, $e$. The heights of these points are:

$$
\begin{aligned}
a & \rightarrow t_{1}^{-}=t_{6}^{-}, \\
b & \rightarrow t_{2}^{+}=t_{3}^{+}, \\
c & \rightarrow t_{3}^{-}=t_{5}^{-}, \\
d & \rightarrow t_{5}^{+}=t_{6}^{+}, \\
e & \rightarrow t_{1}^{+}=t_{4}^{+}=t_{2}^{-}=t_{5}^{-} .
\end{aligned}
$$

They are depicted in fig. 8. To obtain all contributions we divide the circle which represents the singular knot in six parts taking into account that the double point can be regarded as a maximum where the curves $k^{1}$ and $k^{4}$ join, and a minimum where, similarly, the curves $k^{2}$ and $k^{5}$ get attached. The resulting diagram has been depicted in fig. 9. Notice that a dashed line has been drawn to distinguish the points which correspond to the double point. 

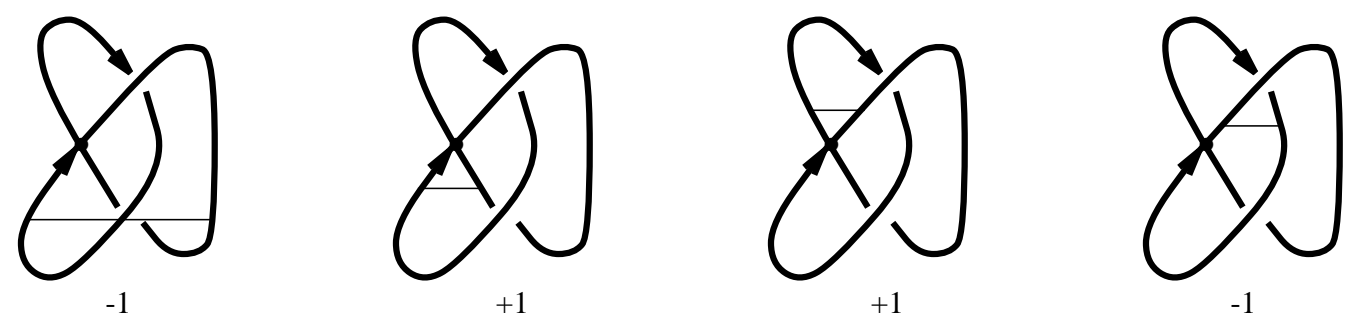

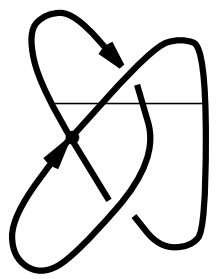

$-1$

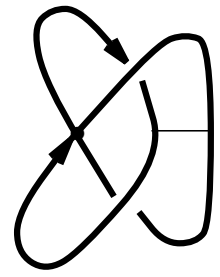

$+1$

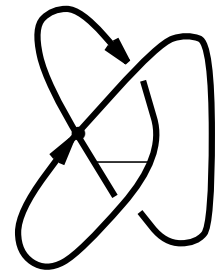

$-1$

Figure 11: Contributions corresponding to a selected group factor.

Also notice that a sign has been assigned to each section of the circle. A plus indicates that the ascending direction coincides with the knot direction. A minus indicates the opposite.

The contributions at order $q=1$ are obtained from the diagrams resulting after joining the curves with a propagator. The group factor for a given choice is obtained computing the trace which emerges after traveling through the circle. To be specific, let us write the contributions corresponding to the group factor $\operatorname{Tr}\left(T^{a} T^{b} T^{a} T^{b}\right)$. The possible contributions are encoded in the diagrams presented in fig. 10. In writing out all these contributions one must take into account that due to the form of the propagator (4.5), a line can not be attached to two sections of the circle which are adjacent to a dashed line. The contributions are easily depicted in the singular knot itself. For each contribution one must compute a sign which is due to the product of the $p_{i j}$ in (4.5). This sign is easily computed using the signs introduced for the sections of the circle in fig. 9. The resulting signs, as well as the singular knot diagrams are pictured in fig. 11. We will assume that the knot is located almost entirely on a plane parallel to the time-axis. Only in a small neighborhood of the crossings the singular knot gets slightly off the plane. The integral corresponding to each contribution is easily obtained with the 
help of the general formula (4.17). For the first diagram one finds:

$$
-(2 x)^{2} \frac{1}{2 \pi i} \frac{1}{2} \int_{t_{3}^{-}}^{t_{1}^{+}} d s\left(\frac{\dot{z}_{1}(s)-\dot{z}_{3}^{\prime}(s)}{z_{1}(s)-z_{3}^{\prime}(s)}+\frac{\dot{z}_{1}^{\prime}(s)-\dot{z}_{3}(s)}{z_{1}^{\prime}(s)-z_{3}(s)}\right) .
$$

This contribution is finite and framing independent so one can take the zerowidth limit before performing the integral. One finds:

$$
-(2 x)^{2} \frac{1}{2 \pi i} \log \left(\frac{z_{1}\left(t_{1}^{+}\right)-z_{3}\left(t_{1}^{+}\right)}{z_{1}\left(t_{3}^{-}\right)-z_{3}\left(t_{3}^{-}\right)}\right) .
$$

The contributions from the fourth to seventh diagrams shown in fig. 11 can be obtained in the same way leading, respectively, to:

$$
\begin{aligned}
& -(2 x)^{2} \frac{1}{2 \pi i} \log \left(\frac{z_{2}\left(t_{2}^{+}\right)-z_{6}\left(t_{2}^{+}\right)}{z_{2}\left(t_{2}^{-}\right)-z_{6}\left(t_{2}^{-}\right)}\right)-(2 x)^{2} \frac{1}{2}, \\
& -(2 x)^{2} \frac{1}{2 \pi i} \log \left(\frac{z_{5}\left(t_{3}^{+}\right)-z_{3}\left(t_{3}^{+}\right)}{z_{5}\left(t_{5}^{-}\right)-z_{3}\left(t_{5}^{-}\right)}\right), \\
& +(2 x)^{2} \frac{1}{2 \pi i} \log \left(\frac{z_{3}\left(t_{3}^{+}\right)-z_{6}\left(t_{3}^{+}\right)}{z_{3}\left(t_{3}^{-}\right)-z_{6}\left(t_{3}^{-}\right)}\right), \\
& -(2 x)^{2} \frac{1}{2 \pi i} \log \left(\frac{z_{4}\left(t_{4}^{+}\right)-z_{6}\left(t_{4}^{+}\right)}{z_{4}\left(t_{4}^{-}\right)-z_{6}\left(t_{4}^{-}\right)}\right)-(2 x)^{2} \frac{1}{2} .
\end{aligned}
$$

Notice that in the first and the fourth expressions of (4.21) we have taken into account the half turn occurring in the fourth and seventh diagrams. In the remaining two contributions one must be careful with the regularization since each one is divergent in the zero-width limit. From the second diagram we have,

$$
\begin{aligned}
& (2 x)^{2} \frac{1}{2 \pi i} \frac{1}{2} \int_{t_{4}^{-}}^{t_{4}^{+}} d s\left(\frac{\dot{z}_{1}(s)-\dot{z}_{4}^{\prime}(s)}{z_{1}(s)-z_{4}^{\prime}(s)}+\frac{\dot{z}_{1}^{\prime}(s)-\dot{z}_{4}(s)}{z_{1}^{\prime}(s)-z_{4}(s)}\right) \\
& =(2 x)^{2} \frac{1}{2 \pi i} \log \left(\frac{\left(z_{1}\left(t_{4}^{+}\right)-z_{4}^{\prime}\left(t_{4}^{+}\right)\right)\left(z_{1}^{\prime}\left(t_{4}^{+}\right)-z_{4}\left(t_{4}^{+}\right)\right)}{\left(z_{1}\left(t_{4}^{-}\right)-z_{4}^{\prime}\left(t_{4}^{-}\right)\right)\left(z_{1}^{\prime}\left(t_{4}^{-}\right)-z_{4}\left(t_{4}^{-}\right)\right)}\right),
\end{aligned}
$$

Similarly, the contribution from the third diagram is:

$$
(2 x)^{2} \frac{1}{2 \pi i} \log \left(\frac{\left(z_{2}\left(t_{2}^{+}\right)-z_{5}^{\prime}\left(t_{2}^{+}\right)\right)\left(z_{2}^{\prime}\left(t_{2}^{+}\right)-z_{5}\left(t_{2}^{+}\right)\right)}{\left(z_{2}\left(t_{2}^{-}\right)-z_{5}^{\prime}\left(t_{2}^{-}\right)\right)\left(z_{2}^{\prime}\left(t_{2}^{-}\right)-z_{5}\left(t_{2}^{-}\right)\right)}\right) .
$$


Adding up (4.22) and (4.23) one gets a quantity which is finite in the zerowidth limit:

$$
(2 x)^{2} \frac{1}{2 \pi i} \log \left(\frac{z_{2}\left(t_{2}^{+}\right)-z_{5}\left(t_{2}^{+}\right)}{z_{1}\left(t_{4}^{-}\right)-z_{4}\left(t_{4}^{-}\right)}\right) .
$$

Summing all the contributions one finds a pairwise cancellation obtaining finally, after putting back the group factor,

$$
-(2 x)^{2} \operatorname{Tr}\left(T^{a} T^{b} T^{a} T^{b}\right)=(2 x)^{2}\left(\frac{1}{2} \sum_{i} \epsilon_{i}\right) \operatorname{Tr}\left(T^{a} T^{b} T^{a} T^{b}\right),
$$

where $\epsilon_{i}, i=1,2$, are the signs at each of the crossings of one of the sections of the singular knot with the other section. This result is a particular case of the general one which will be presented in the next section for Vassiliev invariants of order $n$ corresponding to singular knots with $n-1$ double points.

The cancellation of the singularities at the double point suggests the following equivalent picture in which the framing is not taken into account to avoid the divergences. The idea is to avoid those divergences introducing a principal value prescription. Let us consider a double point as the one in fig. 8 but forgetting about the companion knot and let us introduce a small positive quantity $\eta$. The sum of the two divergent integrals are regularized in the following form:

$$
\int_{t<}^{t^{*}-\eta} d s \frac{\dot{z}_{i}(s)-\dot{z}_{j}(s)}{z_{i}(s)-z_{j}(s)} p_{i j}+\int_{t^{*}+\eta}^{t>} d s \frac{\dot{z}_{i+1}(s)-\dot{z}_{j+1}(s)}{z_{i+1}(s)-z_{j+1}(s)} p_{i+1, j+j} .
$$

This sum is finite in the limit $\eta \rightarrow 0$ and leads to the same result as the one obtained regularizing using the framing. This picture might result more useful in practical calculations. 


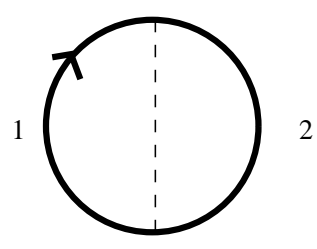

Figure 12: The only configuration for singular knots with one double point.

\section{$5 \quad$ Vassiliev invariants of order $n$ for singular knots with $n-1$ double points}

Another direct application of the operators (1.1) is the derivation of the Vassiliev invariants of order $n$ for singular knots with $n-1$ double points. The result allows to fill very easily the next to top row of an actuality table.

Let us consider a singular knot $K^{n-1}$ with $n-1$ double points. With the help of the operators (1.1) one has that the Vassiliev invariants of order $n$ are given by the following vacuum expectation value:

$$
\begin{aligned}
v_{n}\left(K^{n-1}\right)=(2 x)^{n-1}\left\langle\operatorname { T r } \left[ T^{\phi\left(w_{1}\right)} U\left(w_{1}, w_{2}\right) T^{\phi\left(w_{2}\right)} U\left(w_{2}, w_{3}\right) T^{\phi\left(w_{3}\right)} \ldots\right.\right. \\
\left.\left.\cdots U\left(w_{2 n-3}, w_{2 n-2}\right) T^{\phi\left(w_{2 n-2}\right)} U\left(w_{2 n-2}, w_{1}\right)\right]\right\rangle\left.\right|_{x^{1}}
\end{aligned}
$$

where the subindex $x^{1}$ indicates that one must keep only the terms linear in $x$. To compute (5.1) we must perform the perturbative analysis of the theory. We will carry out the analysis in the light-cone gauge using the construction presented in the previous section. The same result is easily obtained in other gauges.

The simplifying feature of the first order analysis is that there are only contributions from the crossings. This has been shown explicitly in the example considered in the previous section but it is general. Actually, it also holds in other gauges like the Landau gauge. The simplest way to see this in that gauge is by considering the flat knot limit introduced in [29. The first order contribution is therefore very simple, one just has to count how many crossing sings contribute to each group factor.

The analysis of the previous section indicates that we must consider the 
singular knot as the union of a series of curves $k^{i}$ which join extrema and double points. We observed a cancellation of the contributions at the end points of these curves leaving only a part which counts the crossings with signs. This will occur in general and since at the extrema there are no insertions of generators it is most convenient to consider the singular knot as a union of sections $l^{j}, j=1, \ldots, 2(n-1)$, for a knot with $n-1$ double points. The reason for this is that contributions corresponding to a propagator joining points of two different sections, or points of a section with itself have a constant group factor as the end-points move along. The contribution originated from a propagator joining sections $l^{j}$ and $l^{j^{\prime}}$ is

$$
\begin{aligned}
(2 x)^{n} \frac{1}{2} \mathcal{L}\left(j, j^{\prime}\right) \operatorname{Tr}\left[T^{\phi\left(w_{1}\right)} T^{\phi\left(w_{2}\right)}\right. & \cdots T^{\phi\left(w_{j}\right)} T^{a} T^{\phi\left(w_{j+1}\right)} \cdots \\
\cdots & \left.\cdots T^{\phi\left(w_{j^{\prime}}\right)} T^{a} T^{\phi\left(w_{j^{\prime}+1}\right)} \cdots T^{\phi\left(w_{2 n-2}\right)}\right],
\end{aligned}
$$

where $\mathcal{L}\left(j, j^{\prime}\right)$ is the linking number of the section $j$ with section $j^{\prime}$,

$$
\mathcal{L}\left(j, j^{\prime}\right)=\sum_{i} \epsilon_{i},
$$

being the signs $\epsilon_{i}$ the ones in fig. 团, and the sum runs over all the crossings of section $j$ with section $j^{\prime}$. In the case that the end-points of the propagator are attached to the same section of the singular knot one finds a similar contribution:

$$
(2 x)^{n} \frac{1}{2} \mathcal{L}(j, j) \operatorname{Tr}\left[T^{\phi\left(w_{1}\right)} T^{\phi\left(w_{2}\right)} \cdots T^{\phi\left(w_{j}\right)} T^{a} T^{a} T^{\phi\left(w_{j+1}\right)} \cdots T^{\phi\left(w_{2 n-2}\right)}\right],
$$

where $\mathcal{L}$ has the same form as in the other case,

$$
\mathcal{L}(j, j)=\sum_{i} \epsilon_{i},
$$

being now the signs $\epsilon_{i}$ the ones corresponding to the crossings of the section $j$ with itself. Using (5.3) and (5.5) one easily obtains the general form for $v_{n}\left(K^{n-1}\right)$ :

$$
\begin{array}{r}
v_{n}\left(K^{n-1}\right)=(2 x)^{n} \frac{1}{2} \sum_{j \leq j^{\prime}} \mathcal{L}\left(j, j^{\prime}\right) \operatorname{Tr}\left[T^{\phi\left(w_{1}\right)} T^{\phi\left(w_{2}\right)} \cdots T^{\phi\left(w_{j}\right)} T^{a} T^{\phi\left(w_{j+1}\right)} \cdots\right. \\
\left.\cdots T^{\phi\left(w_{j^{\prime}}\right)} T^{a} T^{\phi\left(w_{j^{\prime}+1}\right)} \cdots T^{\phi\left(w_{2 n-2}\right)}\right],
\end{array}
$$



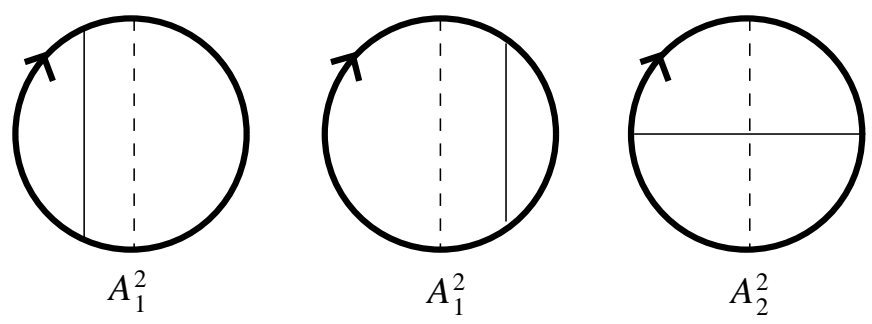

Figure 13: Diagrams corresponding to the contributions to $v_{2}\left(K^{1}\right)$.

where it is understood that for $j=j^{\prime}$ this expression takes the form (5.4).

To illustrate the content of (5.6) we will present its explicit form for $n=2,3$. For the case $n=2$ we must consider singular knots with one double point. There is only one possible configuration in this case. It is pictured in fig. 12. In order to obtain $v_{2}\left(K^{1}\right)$ as given in (5.6) we must insert in the diagram shown in fig. 12 a propagator in all possible ways. The three inequivalent possibilities are shown in fig. 13. The possible group factors at second order are:

$$
A_{1}^{2}=\operatorname{Tr}\left(T^{a} T^{a} T^{b} T^{b}\right), \quad A_{2}^{2}=\operatorname{Tr}\left(T^{a} T^{b} T^{a} T^{b}\right) .
$$

Below each contribution shown in fig. 13 the corresponding group factor is shown. The resulting form for $v_{2}\left(K^{1}\right)$ is:

$$
v_{2}\left(K^{1}\right)=2 x^{2}\left[(\mathcal{L}(1,1)+\mathcal{L}(2,2)) A_{1}^{2}+\mathcal{L}(1,2) A_{2}^{2}\right] .
$$

Before entering into the discussion of the Vassiliev invariants of order three we will make a simple check of the result (5.8). Using the Lie-algebra relations of the generators, the expression (5.8) can be written in terms of the group factors of a canonical basis [34]:

$$
v_{2}\left(K^{1}\right)=2 x^{2} w\left(K^{1}\right) \operatorname{Tr}\left(T^{a} T^{a} T^{b} T^{b}\right)+2 x^{2} \mathcal{L}(1,2) f_{a b c} \operatorname{Tr}\left(T^{a} T^{b} T^{c}\right),
$$

where $w\left(K^{1}\right)$ is the writhe of the singular knot (the double point does not contribute to $\left.w\left(K^{1}\right)\right)$.

The result (5.9) can be verified very simply using the explicit form for $v_{2}\left(K^{0}\right)$ given in [29]. In that paper it was shown that:

$$
v_{2}\left(K^{0}\right)=\frac{1}{2} x^{2} w\left(K^{0}\right)^{2} \operatorname{Tr}\left(T^{a} T^{a} T^{b} T^{b}\right)+x^{2}\left(\rho_{2}\left(K^{0}\right)+\rho_{1}\left(K^{0}\right)\right) f_{a b c} \operatorname{Tr}\left(T^{a} T^{b} T^{c}\right),
$$




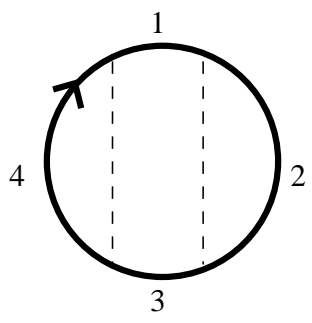

$a$

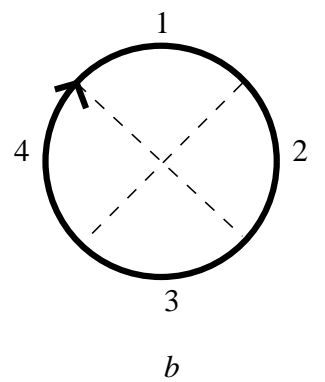

Figure 14: Inequivalent configurations of a singular knot with two double points.

where,

$$
\rho_{2}\left(K^{0}\right)=\sum_{(j>i)} \epsilon_{i} \epsilon_{j},
$$

being $\epsilon_{i}$ and $\epsilon_{j}$ the signatures of the crossings $i$ and $j$ of $K^{0}$, respectively. By $(j>i)$ we mean pairs of crossings $i, j$ such that $s_{i}<s_{j}<t_{i}<t_{j}$. In (5.10), $w\left(K^{0}\right)$ is the writhe of $K^{0}$ and $\rho_{1}\left(K^{0}\right)$ is a quantity which satisfies:

$$
\rho_{1}\left(K_{+}^{0}\right)-\rho_{1}\left(K_{-}^{0}\right)=0
$$

where $K_{+}^{0}$ and $K_{-}^{0}$ differ by the exchange of an overcrossing by an undercrossing. Using (5.11) and (5.12), as well as the relations,

$$
\begin{aligned}
w\left(K_{+}^{0}\right)^{2}-w\left(K_{-}^{0}\right)^{2} & =4 w\left(K^{1}\right), \\
\rho_{2}\left(K_{+}^{0}\right)-\rho_{2}\left(K_{-}^{0}\right) & =2 \mathcal{L}(1,2),
\end{aligned}
$$

one easily proves that, indeed, $v_{2}\left(K_{+}^{0}\right)-v_{2}\left(K_{-}^{0}\right)$ corresponds to the expression obtained in (5.9).

We will end this section presenting the explicit form of the third-order Vassiliev invariant for knots with two double points, $v_{3}\left(K^{2}\right)$. In this case there are two inequivalent configurations of $K^{2}$. They are presented in fig. 14. We will denote by $K_{a}^{2}\left(K_{b}^{2}\right)$ a choice of singular knot for the configuration $a(b)$. Our aim is to compute $v_{3}\left(K_{a}^{2}\right)$ and $v_{3}\left(K_{b}^{2}\right)$ using (5.6). As before, we begin labeling the sections of the singular knots as shown in fig. 14, then we insert one propagator in all possible ways. To each insertion corresponds a group factor. Denoting the possible group factors at order three by:

$$
A_{1}^{3}=\operatorname{Tr}\left(T^{a} T^{a} T^{b} T^{b} T^{c} T^{c}\right),
$$



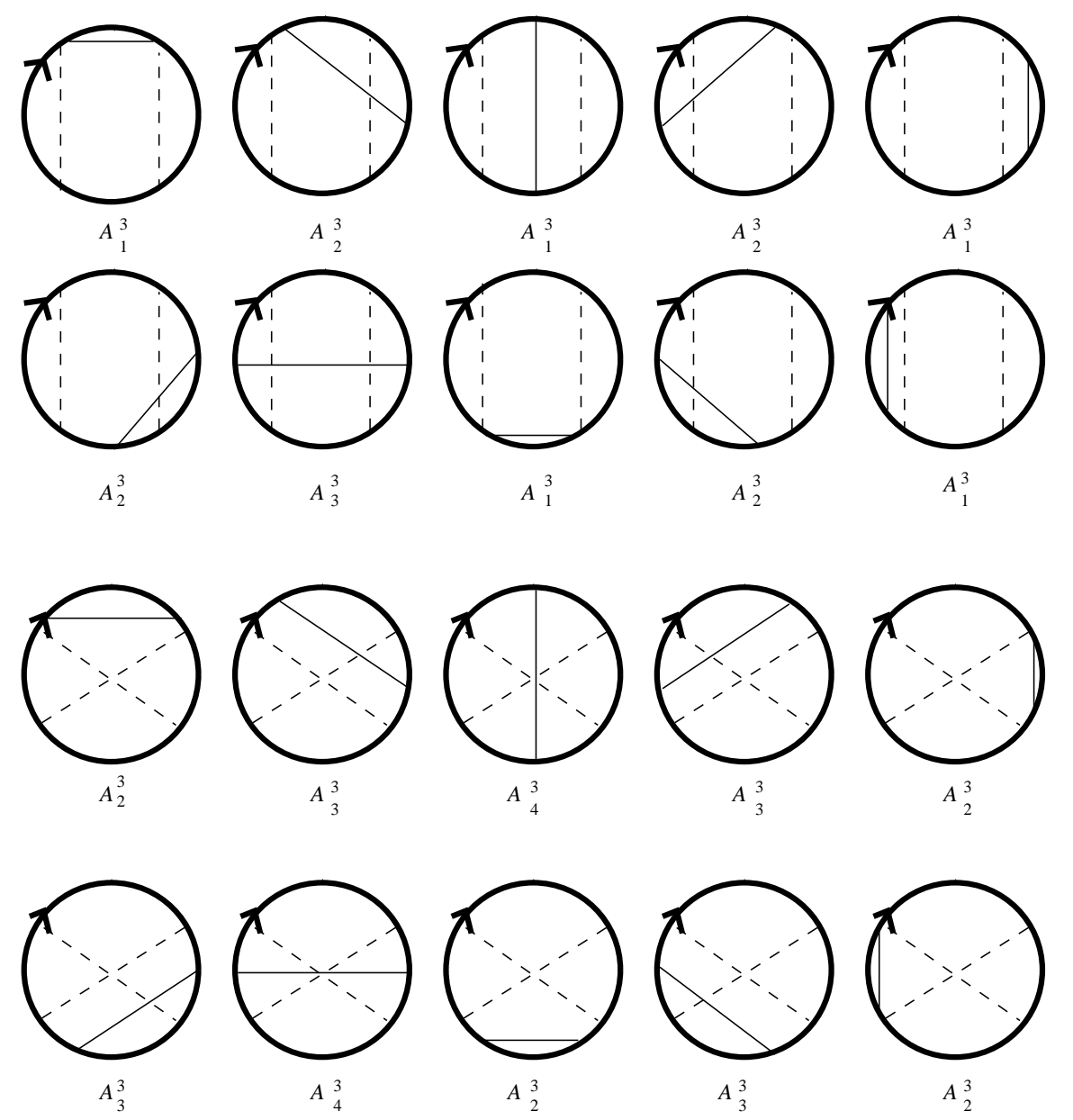

Figure 15: Diagrams corresponding to the contributions to $v_{3}\left(K_{a}^{2}\right)$ and $v_{3}\left(K_{b}^{2}\right)$. 


$$
\begin{aligned}
& A_{2}^{3}=\operatorname{Tr}\left(T^{a} T^{b} T^{a} T^{b} T^{c} T^{c}\right), \\
& A_{3}^{3}=\operatorname{Tr}\left(T^{a} T^{b} T^{c} T^{a} T^{c} T^{b}\right), \\
& A_{4}^{3}=\operatorname{Tr}\left(T^{a} T^{b} T^{c} T^{a} T^{b} T^{c}\right),
\end{aligned}
$$

one easily builds the table shown in fig. 15. With the help of that table and (5.6) one finds:

$$
\begin{aligned}
v_{3}\left(K_{a}^{2}\right)= & 4 x^{3}\left[(\mathcal{L}(1,1)+\mathcal{L}(2,2)+\mathcal{L}(3,3)+\mathcal{L}(4,4)+\mathcal{L}(1,3)) A_{1}^{3}\right. \\
& \left.+(\mathcal{L}(1,2)+\mathcal{L}(1,4)+\mathcal{L}(2,3)+\mathcal{L}(3,4)) A_{2}^{3}+\mathcal{L}(2,4) A_{3}^{3}\right]
\end{aligned}
$$

and,

$$
\begin{aligned}
& v_{3}\left(K_{b}^{2}\right)=4 x^{3}\left[(\mathcal{L}(1,1)+\mathcal{L}(2,2)+\mathcal{L}(3,3)+\mathcal{L}(4,4)) A_{2}^{3}\right. \\
& \left.+(\mathcal{L}(1,2)+\mathcal{L}(1,4)+\mathcal{L}(2,3)+\mathcal{L}(3,4)) A_{3}^{3}+(\mathcal{L}(1,3)+\mathcal{L}(2,4)) A_{4}^{3}\right]
\end{aligned}
$$

The result (5.6) allows to complete in a very simple form the next to the top row of an actuality table. In fig. 16 we have shown some elements of the actuality tables corresponding to $n=2,3$. Recall that the top row is given by the form of the operators (11.1) at order zero. In the case of $n=2$ the table shown is the complete table. Notice that in this case the two elements of the top row are independent. For $n=3$ only three of the four elements shown in top row are independent. For the next to the top row we have chosen some representatives corresponding to the two possible configurations and we have computed (5.15) and (5.16) for each of them. The results are depicted in the right lower corners. The next to the top row corresponding to higher values of $n$ can be obtained similarly. 

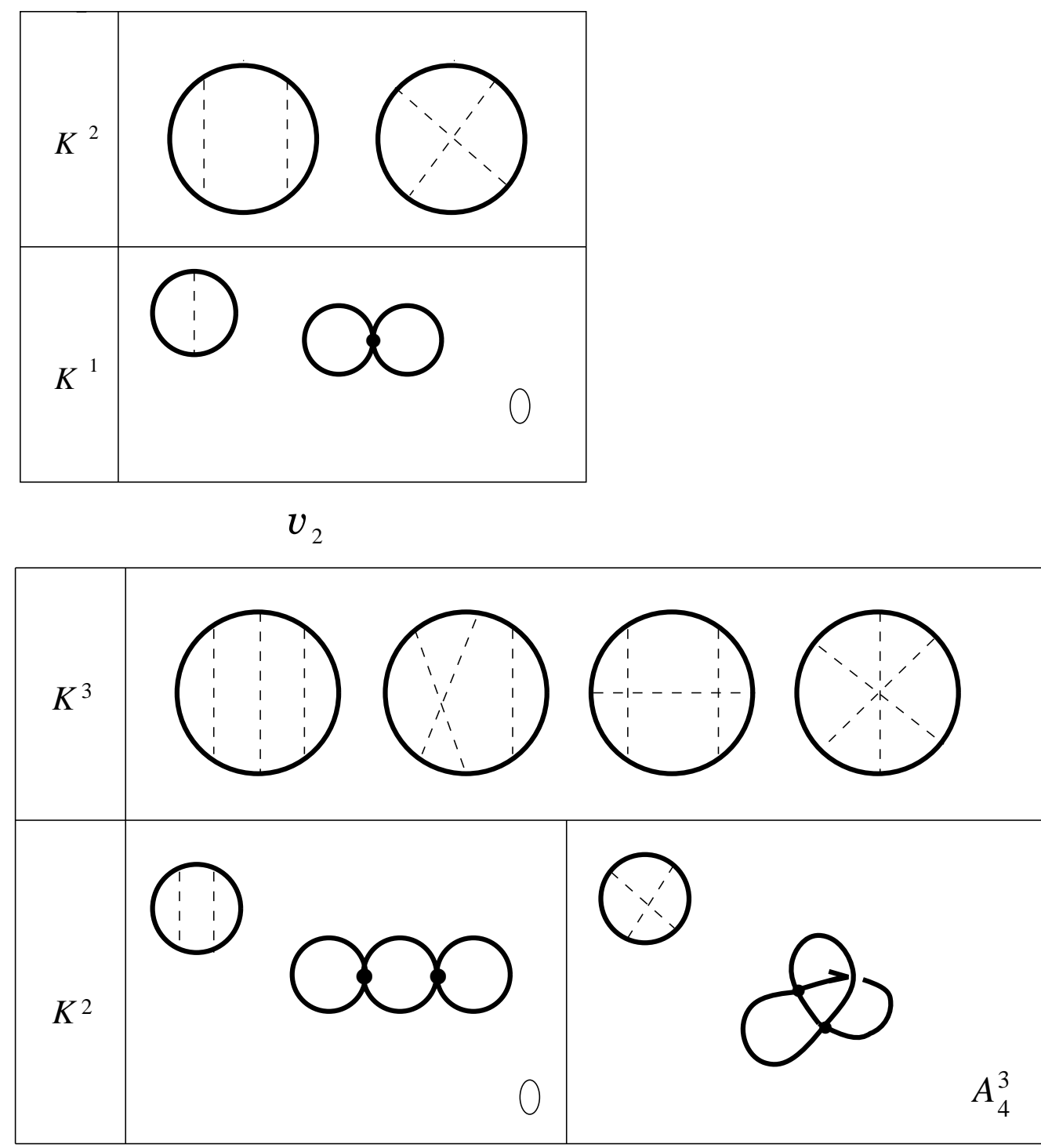

$v_{3}$

Figure 16: Elements of the actuality table for $v_{2}$ and $v_{3}$. 


\section{Conclusions}

In this paper we have constructed the natural operators in the context of Chern-Simons gauge theory to compute invariants associated to singular knots. These operators have a very simple structure and consists of the trace of an ordered series of Wilson lines, which are associated to each of the sections of the singular knot, with adequate insertions of group generators at the double points. They lead naturally to the association of configurations to singular knots in the standard way in which it is done in knot theory. These operators allow to obtain simple proofs of the Birman and Lin theorem for the expansion of a polynomial invariant, and Bar-Natan's theorem for the integrability of weight systems. Actually, our work constitutes a gauge independent (though field-theory based) proof of this last theorem.

The analysis of Chern-Simons gauge theory in the light-cone gauge has led to an expression for the vacuum expectation value of the operators (1.1) which constitutes a generalization of the Knotsevich integral to the case of framed singular knots. We have shown the finiteness of this expression and we have computed it explicitly at lowest order. This last result has provided the necessary information to obtain the general form of the Vassiliev invariant of order $n$ for singular knots with $n-1$ double points. We have shown how this expression allows to construct very simply the next to the top row of an actuality table.

Our work opens a variety of investigations. One important issue consists of the analysis of the operators (1.1) from a non-perturbative point of view. Methods as the one used in [2, 3, 5, 6] should be applied to obtain information on the invariants associated to singular knots.

The study of the operators (1.1) from a perturbative point of view should be pursued further. For example, it would be very interesting to work out the structure of the perturbative series expansion associated to the vacuum expectation values of the operators (1.1) in covariant gauges. But perhaps the most important issue that one should address within the perturbative approach is if the operators (1.1) are enough powerful to provide relations among Vassiliev invariants which could lead to a combinatorial formula for these invariants, the simplest case being the one obtained for $v_{n}\left(K^{n-1}\right)$. These and other related issues will be addressed in future work. 


\section{Acknowledgements}

This work was supported in part by DGICYT under grant PB93-0344, and by the EU Commission under TMR grant FMAX-CT96-0012.

\section{References}

[1] E. Witten, Commun. Math. Phys. 121 (1989) 351.

[2] M. Bos and V.P. Nair, Phys. Lett. B223 (1989) 61 and Int. J. Mod. Phys. A5 (1990) 959; S. Elitzur, G. Moore, A. Schwimmer and N. Seiberg, Nucl. Phys. B326 (1989) 108.

[3] J.M.F. Labastida and A.V. Ramallo, Phys. Lett. B227 (1989) 92 and B228 (1989) 214; J.M.F. Labastida, P.M. Llatas and A.V. Ramallo, Nucl. Phys. B348 (1991) 651; J.M.F. Labastida and M. Mariño, Int. J. Mod. Phys. A10 (1995) 1045; J.M.F. Labastida and E. Pérez, J. Math. Phys. 37 (1996) 2013.

[4] J. Frohlich and C. King, Commun. Math. Phys. 126 (1989) 167.

[5] S. Martin, Nucl. Phys. B338 (1990) 244.

[6] R.K. Kaul, Commun. Math. Phys. 162 (1994) 289; R.K. Kaul and T.R. Govindarajan, Nucl. Phys. B380 (1992) 293 and B393 (1993) 392; P. Ramadevi, T.R. Govindarajan and R.K. Kaul, Nucl. Phys. B402 (1993) 548; Mod. Phys. Lett. A10 (1995) 1635.

[7] V. F. R. Jones, Bull. AMS 12 (1985) 103; Ann. of Math. 126 (1987) 335.

[8] P. Freyd, D. Yetter, J. Hoste, W.B.R. Lickorish, K. Millet and A. Ocneanu, Bull. AMS 12 (1985) 239.

[9] L.H. Kauffman, Trans. Am. Math. Soc. 318 (1990) 417.

[10] Y. Akutsu and M. Wadati, J. Phys. Soc. Jap. 56 (1987) 839 and 56 (1987) 3039. 
[11] E. Guadagnini, M. Martellini and M. Mintchev, Phys. Lett. B227 (1989) 111 and B228 (1989) 489; Nucl. Phys. B330 (1990) 575.

[12] D. Bar-Natan, "Perturbative aspects of Chern-Simons topological quantum field theory", Ph.D. Thesis, Princeton University, 1991.

[13] J.F.W.H. van de Wetering, Nucl. Phys. B379 (1992) 172.

[14] M. Alvarez and J.M.F. Labastida, Nucl. Phys. B395 (1993) 198 and B433 (1995) 555; Erratum, ibid. B441 (1995) 403.

[15] M. Alvarez and J.M.F. Labastida, Journal of Knot Theory and its Ramifications 5 (1996) 779; M. Alvarez, J.M.F. Labastida and E. Pérez, Nucl. Phys. B488 (1997) 677.

[16] D. Altschuler and L. Freidel, Commun. Math. Phys. 187 (1997) 261 and 170 (1995) 41.

[17] J.M.F. Labastida and E. Pérez, "Kontsevich Integral for Vassiliev Invariants from Chern-Simons Perturbation Theory in the Light-Cone Gauge", CERN and Santiago de Compostela preprint, CERN-TH/97-282, USFT-32/97; hep-th/9710176.

[18] B. Bruegmann, R. Gambini and J. Pullin Nucl. Phys. B385 (1992) 587.

[19] B. Bruegmann, Int. J. Theo. Phys. 34 (1995) 145.

[20] R. Gambini and J. Pullin, Commun. Math. Phys. 185 (1997) 621.

[21] J.S. Birman and X.S. Lin, Invent. Math. 111 (1993) 225; J.S. Birman, Bull. AMS 28 (1993) 253.

[22] M. Kontsevich, Advances in Soviet Math. 16, Part 2 (1993) 137.

[23] T.Q.T. Le and J. Murakami, "The universal Vassiliev-Kontsevich invariant for framed oriented links", Max-Plank Institut fur Mathematik preprint, 1994.

[24] D. Bar-Natan, Topology 34 (1995) 423.

[25] V.G. Knizhnik and A.B. Zamolodchikov, Nucl. Phys. B247 (1984) 83. 
[26] G. Leibbrandt and C.P. Martin, Nucl. Phys. B377 (1992) 593 and B416 (1994) 351.

[27] A. Brandhuber, S. Emery, M. Langer, O. Piguet, M. Schweda and S.P. Sorella, Helv. Phys. Acta 66 (1993) 551.

[28] G. Leibbrandt, Rev. Mod. Phys. 59 (1987) 1067.

[29] A.C. Hirshfeld and U. Sassenberg, Journal of Knot Theory and its Ramifications 5 (1996) 489 and 5 (1996) 805.

[30] A.S. Cattaneo, P. Cotta-Ramusino, J. Frohlich and M. Martellini, J. Math. Phys. 36 (1995) 6137.

[31] L. Alvarez-Gaumé, J.M.F. Labastida and A. V. Ramallo, Nucl. Phys. B334 (1990) 103; G. P. Korchemsky, Mod. Phys. Lett. A6 (1991) 727.

[32] A. Blasi, O. Piguet and S.P. Sorella, Nucl. Phys. B356 (1991) 154.

[33] C. P. Martin, Phys. Lett. B241 (1990) 513; G. Giavarini, C.P. Martin, F. Ruiz Ruiz, Nucl. Phys. B381 (1992) 222.

[34] M. Alvarez and J.M.F. Labastida, Commun. Math. Phys. 189 (1997) 641. 